\title{
PAV ontology: provenance, authoring and versioning
}

\author{
Paolo Ciccarese ${ }^{1,2^{*}+}$, Stian Soiland-Reyes ${ }^{3+}$, Khalid Belhajjame ${ }^{3}$, Alasdair JG Gray ${ }^{3}$, Carole Goble ${ }^{3}$ and Tim Clark ${ }^{1,2,3}$
}

\begin{abstract}
Background: Provenance is a critical ingredient for establishing trust of published scientific content. This is true whether we are considering a data set, a computational workflow, a peer-reviewed publication or a simple scientific claim with supportive evidence. Existing vocabularies such as Dublin Core Terms (DC Terms) and the W3C Provenance Ontology (PROV-O) are domain-independent and general-purpose and they allow and encourage for extensions to cover more specific needs. In particular, to track authoring and versioning information of web resources, PROV-O provides a basic methodology but not any specific classes and properties for identifying or distinguishing between the various roles assumed by agents manipulating digital artifacts, such as author, contributor and curator.

Results: We present the Provenance, Authoring and Versioning ontology (PAV, namespace http://purl.org/pav/): a lightweight ontology for capturing "just enough" descriptions essential for tracking the provenance, authoring and versioning of web resources. We argue that such descriptions are essential for digital scientific content. PAV distinguishes between contributors, authors and curators of content and creators of representations in addition to the provenance of originating resources that have been accessed, transformed and consumed. We explore five projects (and communities) that have adopted PAV illustrating their usage through concrete examples. Moreover, we present mappings that show how PAV extends the W3C PROV-O ontology to support broader interoperability.

Method: The initial design of the PAV ontology was driven by requirements from the AlzSWAN project with further requirements incorporated later from other projects detailed in this paper. The authors strived to keep PAV lightweight and compact by including only those terms that have demonstrated to be pragmatically useful in existing applications, and by recommending terms from existing ontologies when plausible.

Discussion: We analyze and compare PAV with related approaches, namely Provenance Vocabulary (PRV), DC Terms and BIBFRAME. We identify similarities and analyze differences between those vocabularies and PAV, outlining strengths and weaknesses of our proposed model. We specify SKOS mappings that align PAV with DC Terms. We conclude the paper with general remarks on the applicability of PAV.
\end{abstract}

Keywords: Provenance, Authoring, Versioning, Annotation, Semantic web, Attribution

\section{Background}

Research in the life sciences is becoming increasingly digital and collaborative. Scientists tend to conduct their investigations and reporting using digital resources (e.g., data artifacts, articles, etc.) obtained by aggregating existing resources (potentially generated as a result of other research investigations conducted by other

\footnotetext{
*Correspondence: paolo.ciccarese@gmail.com

${ }^{\dagger}$ Equal contributors

'Department of Neurology, Massachusetts General Hospital, 55 Fruit Street, Boston, MA 02114, USA

${ }^{2}$ Harvard Medical School, 25 Shattuck Street, Boston, MA 02115, USA

Full list of author information is available at the end of the article
}

scientists), and processed and analyzed using manual or automated workflows.

In such a context, scientists require a systematic means to organize and annotate resources [1]. This might require them, amongst other things, to: (i) trace the origin of a given resource; (ii) specify its previous and subsequent versions; and (iii) identify the creators (be they humans or machines) responsible for the existence of the resource, as well as the contributors who enriched and updated its content. For the most general use, a provenance vocabulary that meets these criteria must also be relatively compact and terse.

\section{Biomed Central}


Several provenance vocabularies including Dublin Core Terms (DC Terms) [2], PROV-O [3], OPM [4], and Provenance Vocabulary [5] partially address these general needs, at varying levels of richness, complexity and maturity. In this section, after discussing the original use case, we discuss the strengths and weaknesses of these vocabularies and gaps in usage coverage, which led us to develop the Provenance Authoring and Versioning (PAV) ontology. In the Results section we also provide mappings from PAV to PROV-O and DC Terms.

\section{Original use case: SWAN platform}

The Semantic Web Applications in Neuromedicine (SWAN) web-based collaborative platform [6] is an example of an application that embodies many of the above requirements. SWAN aims to organize and annotate scientific knowledge regarding neurodegenerative disorders and to facilitate the formation, development and testing of hypotheses. In particular, the AlzSWAN [7] knowledge base (AlzSWAN KB), a collaboration of SWAN's developers with the AlzForum web community of Alzheimer Disease researchers [8], is an instance of SWAN configured to allow the scientific community of Alzheimer Disease (AD) researchers to author, curate and connect a diversity of data and ideas about AD. The AlzSWAN curators typically read carefully a scientific article, usually representing a hypothesis on $\mathrm{AD}$, and produce a linear representation of the embedded scientific discourse: claims, hypotheses and questions. For each of the discourse elements the curator selects related publications, proteins and genes. Knowledge in the AlzSWAN KB is shared using the SWAN Ontology [9] for interoperability.

One of the goals of the AlzSWAN KB consists in clearly recording the provenance of the digital artifacts as well as the provenance of the content or knowledge elements represented by the artifacts, and the agents (organizations, people and software) involved in creating and manipulating those artifacts. There is a clear distinction between the roles of the authors and curators, and the source of content:

Authors are the primary originators of scientific statements, originally conceiving the content (e.g. a tabular dataset).

Curators collect the knowledge published by the authors, interpreting and transforming the content of a textual document into SWAN research statements (hypothesis, claim or research question). They restructure the previously authored content and shape it to be appropriate for the intended representation (e.g. by normalizing the fields for being represented in a spreadsheet). Curators create the SWAN KB version that embodies the authors' work; thus they are contributing to the knowledge representation. However, the main intellectual property remains attributed to the original authors.

Artifact creators take care of physically creating the digital artifact by entering the statements and their links into the platform, (e.g. saves the spreadsheet as an $x l s x$ file).

External sources are the external data- and knowledgebases such as PubMed [10] and UniProt [11,12] that AlzSWAN draws upon for metadata and for integrated data. Some of this metadata are retrieved and cached as they are, while some are imported after one or more transformations. It is important to track the original source and how it was incorporated in the knowledge base.

As depicted in Figure 1, the AlzSWAN knowledge capture and curation process consists of several steps:

A PhD-level neuroscientist (the curator) reads carefully an article (written by authors) usually representing a hypothesis on AD.

Based on the reading, the curator produces a textual document with a linear knowledge representation of the scientific discourse of the article, by building an ordered list of claims, hypotheses and questions.

For each of those elements, the curator identifies external resources such as related publications, proteins and genes. These resources provide data that can be retrieved in unmodified form, or imported after a transformation.

When possible, the formed representation is shared with the authors of the original article for collecting feedback.

The knowledge map is entered in AlzSWAN by a second person (the artifact creator) through a web user interface, which eventually encodes the textual content according to the SWAN ontology.

While step 5 represents the straightforward creation of the digital artifact, steps 1-4 represent the curation of the knowledge that the authors expressed in the journal article. Curation involves high-level domain knowledge and acts of judgement and creative composition. Both authors and curators originate digital content; authorship denotes the role of creative invention of a work, while the artifact creator of a work in our terminology is responsible for accurate transcription and encoding into final digital form.

Figure 1 also depicts tasks of revision, publishing and feedback collection. In particular, the feedback might motivate the generation of a new version of the encoded knowledge. Normally, in the case of AlzSWAN a new version might include newly available evidence supporting a given claim.

\section{Existing provenance vocabularies}

There are existing vocabularies that at first appear to be promising to address the needs of AlzSWAN and similar 


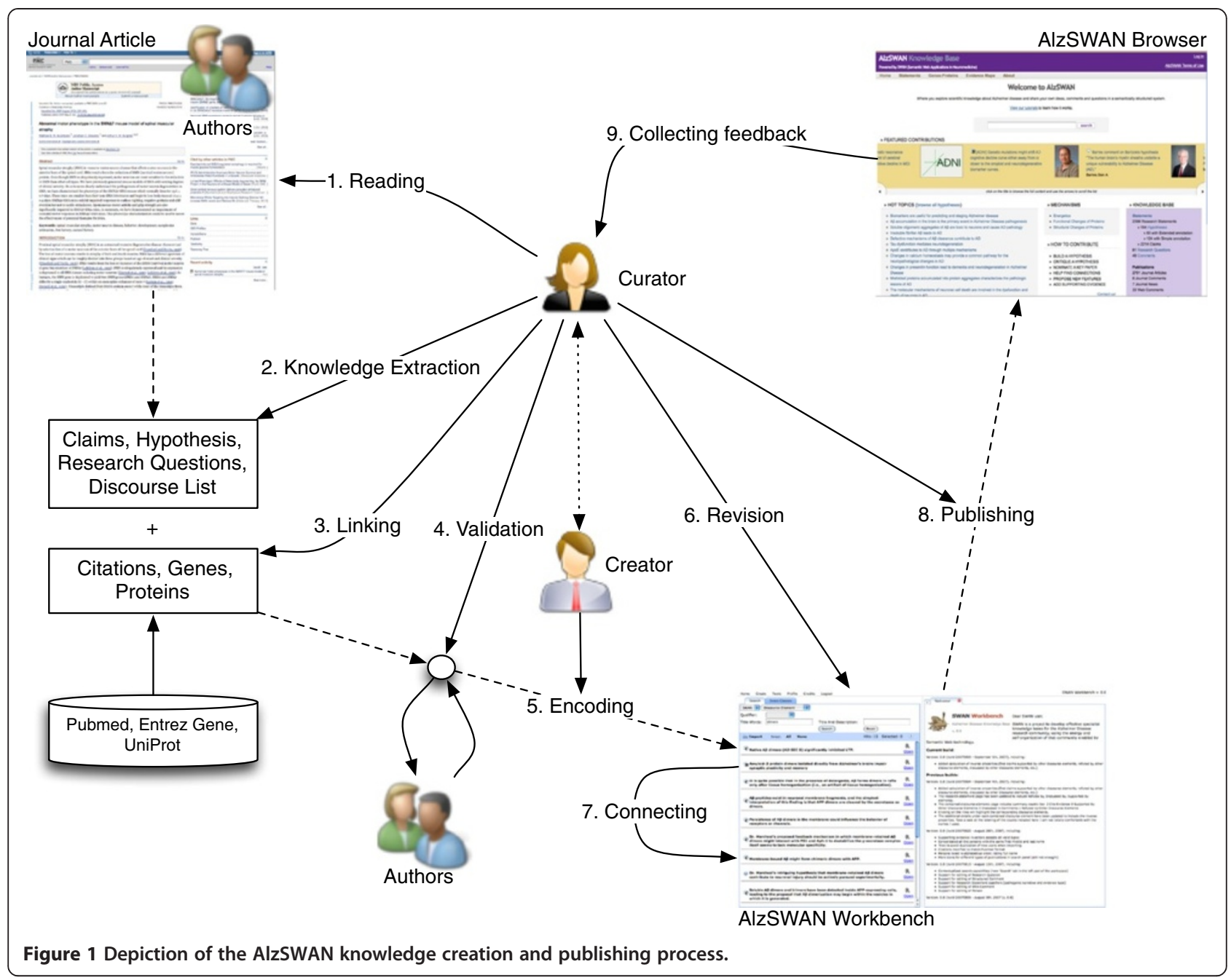

applications: Dublin Core Terms (DC Terms) [2], PROV-O [3], OPM [4], and Provenance Vocabulary [5].

The Dublin Core Metadata Initiative (DCMI) provides core metadata vocabularies to support interoperable solutions for discovering and managing resources. In particular, DC Terms provides terms for specifying the entities that create and/or contribute to the existence of a given resource. While terms such as dct:contributor and dct:creator are useful and popular, we argue that they conflate what we consider as the distinct roles of contributor/author/curator and creator of the representation. For instance, a person who converts a web page from HTML 3 to HTML 5 could be said to be the dct:creator or dct:contributor of the new document, even when that person has not modified the human readable content of the document.

DC Terms provides a means for specifying derivation and representational differences (dct:source, dct: isVersionOf, dct:isFormatOf), but these do not clearly distinguish between a resource which was simply copied, one which was transformed to give rise to a new resource, or one which was further derived by adding additional content (for example new scientific evidence). For versioning DC Terms has dct:isVersion $O f$, which implies substantive changes in content (e.g. a movie can be a version of a theater play), and dct:replaces, which indicates a superseded resource. However, these statements do not quite fit with dealing with and distinguishing between: smaller updates (e.g. spelling mistakes); larger derivations (which might no longer be versions of the original); and linear revision history without necessarily indicating previous versions as outdated. Consider, for example, a health authority where Dr. Doe authored and implemented the clinical guidelines for treating hypertensive patients and sent it to Dr. Green for comments. Green edits his copy to suggest extending a drug treatment from 2 weeks to 4 weeks. Because Green's guideline has not been approved yet, we cannot state that his document replaces (dct:replaces) Doe's document. Also, Green's document is in the same form 
and does not introduce substantive changes (dct: isVersion $O f$ ) from Doe's document.

PROV-O [3], an OWL ontology developed by the W3C Provenance Working group [13] aims to provide a standard for representing and exchanging domainindependent provenance information between applications and systems. PROV-O provides terms that can be used to trace the origin of a given resource, its derivation history, as well as the relationship between the resources, and the entities that contributed to the existence of the resource. PROV-O can be used at detailed process level with activity-agent-entity interactions, or at a higher level with shortcuts for entity-entity and entity-agent relations such as prov:wasDerivedFrom and prov:wasAtributedTo.

Overall PROV-O is a generic provenance data model, which can be extended to give domain-specific provenance, for instance by subclasses of prov:Activity or subproperties of prov:wasAttributedTo. PROV-O does not itself provide any distinctions between authors, curators, contributors or artifact creators.

PROV-O specifies a set of common extensions, which at first glance would seem to cover some of our requirements: i) prov:hadPrimarySource: a kind of "derivation relation from secondary materials to their primary sources, which were produced by some agent with direct experience and knowledge about the topic", ii) prov:wasQuotedFrom, the "repeat of (some or all of) an entity, such as text or image, by someone who may or may not be its original author".

However, on closer examination these terms are inadequate. For example, in the AlzSWAN project, curators consulted papers on the web. Such an action cannot be described using prov:hadPrimarySource, because the documents consulted by the curators were not necessarily primary sources [3] such as witness statements, reports or interviews; we found the definition of primary sources to be too narrow to cover most academic publications. Instead, we need a means to describe the fact that the curators simply accessed a document. Curators can also download a file from a source on the web, such as UniProt. Although prov:wasQuotedFrom can be used to relate the document to the source it was downloaded from, it does not reflect that the document is a complete and exact copy of the document in the source.

The Open Provenance Model [4] predates PROV-O, and has a very similar approach to modeling provenance by relating agents, artifacts and processes. By and large, the concepts of OPM are covered by equivalent PROV$\mathrm{O}$ concepts, therefore the above analysis of PROV-O applies also to OPM.

DC Terms, PROV-O and OPM are domain-independent and general-purpose vocabularies. Therefore the limitations we identified above should not be perceived as issues that need to be addressed within these vocabularies. In fact, the PROV-O authors do not claim that the vocabulary is complete. Instead, they encourage users to extend it with terms that capture their domain needs.

The Provenance Vocabulary [5] describes data access, creation, retrieval and publishing as detailed chains of prov: Activity and prov:Entity relations. Terms like prv:accessedResource, prv:createdBy, prv:retrievedBy seem relevant for our use cases, but as detailed in the Discussion section, this approach come at the cost of increased verbosity, which we argue reduces the ability to query the provenance in a consistent way.

For describing the provenance of the AlzSWAN use cases such as the one depicted in Figure 1, we designed the PAV (Provenance, Authoring and Versioning) ontology, whose most recent version, PAV 2, maps to PROV-O. In PAV, we do not attempt to model the whole chain of process-oriented provenance like Open Provenance Model (OPM) processes [4] and PROV-O activities [3], or the many forms of metadata as covered by Dublin Core Terms (DC Terms) [2]. Rather, in PAV we focus on the provenance of a digital resource in terms of its relationships with other digital resources and agents involved in their creation, authoring and manipulation, and we abstract away from the description of the activities (process) that manipulate and transform the digital resources.

\section{Results}

In this section we present the PAV ontology, describing its structure and constituent terms. We go on to present the systems and communities that have adopted PAV, and to discuss how they use it. Finally, we present a collection of mappings, specifying how PAV extends the W3C PROV-O vocabulary.

\section{PAV Ontology}

PAV is a lightweight vocabulary, for capturing "just enough" descriptions essential for web resources representing digitized knowledge. PAV is intended to specify Provenance, Authoring and Versioning information. Accordingly, this section is organized into three subsections. PAV properties are outlined in bold where they are defined, described or exemplified; and in italics elsewhere. Properties belonging to other vocabularies are always in italics.

The pav: prefix indicates the namespace http://purl. org/pav/ [14], which also resolves to the latest version of the PAV ontology as OWL. PAV is currently in version 2.2 (OWL importable from http://purl.org/pav/2.2 [15]). Further details of versions and changes are listed on the wiki [16]. 


\section{Authoring}

In scholarly communication, it is crucial to be able to precisely attribute the several forms of authorship (intellectual property) or contributions of the knowledge content and of its representation [1]. The PAV ontology provides properties for tracking intellectual property information, which are described in Table 1.

As suggested by the properties listed in Table 1, in PAV we distinguish between authors that originate or creatively invent a work that is expressed in a digital resource (pav:authoredBy), e.g., the authors of a scientific publication or of a novel scientific hypothesis; and curators (pav:curatedBy), who are content specialists responsible for shaping the expression in an appropriate format. When talking about knowledge artifacts the authors are contributing the primary knowledge and the curators are those responsible for updating the knowledge base. Contributors (identified by the superproperty pav:contributedBy) cover both authors and curators, as well as agents that generically provide some help in conceiving the resource or in the expressed knowledge creation/extraction. For example, a scientist who performed some biological experiments and published the results in a paper is considered an author (pav:authoredBy) as she produced novel results. The agent that analyzes the paper, extracts and organizes some of the scientific discourse in argumentation - hypotheses, claims, etc. - is the curator (pav:curatedBy). Finally the person that enters such knowledge in a hypotheses management application is the creator of the knowledge artifacts (pav:createdBy), as defined in the next subsection.

As illustrated in Table 1, PAV authoring properties can be associated with a timestamp using the following properties: pav:authoredOn, pav:curatedOn, and pav: contributedOn.

For describing the publication process of the created resource, we recommend adopting the following DC Terms properties: dct:publisher, dct:issued, dct:dateSubmitted, dct:dateAccepted and dct:dateCopyrighted. For instance, dct:publisher identifies 'an entity responsible for making the resource available' [2]. It is important to note that these properties describe the publication of the particular resource (say a knowledge graph), not the publication of the original work that this resource might have been imported or derived from. The PAV term pav:

\section{Table 1 PAV authoring properties}

\begin{tabular}{ll}
\hline pav:authoredBy & Indicates an agent that originated or gave existence to the work that is expressed by the digital resource. The author of the \\
content of a resource may be different from the creator of that resource representation (pav:createdBy), although they are often
\end{tabular} the same.

pav:authoredBy is more specific than its superproperty dct:creator - which might or might not be interpreted to also cover the creation of the representation of the artifact.

The author is usually not a software agent (which would be indicated with pav:createdWith, pav:createdBy or pav:importedBy), unless the software actually authored the content itself; for instance an artificial intelligence algorithm which authored a piece of music or a machine learning algorithm that authored a classification of a tumor sample [17].

pav:authoredOn Indicates the date this resource was authored by the agents given by pav:authoredBy. Note that pav:authoredOn is different from pav:createdOn, although their values are often the same.

This property is normally used in a functional way, indicating the last time of authoring, although PAV does not formally restrict this.

pav:curatedBy Specifies an agent specialist responsible for shaping the expression in an appropriate format. Often the primary agent responsible for ensuring the quality of the representation. The curator may be different from the author (pav:authoredBy) and creator of the digital resource (pav:createdBy). The curator may in some cases be a software agent, for instance text mining software which adds hyperlinks for recognized genome names.

pav:curatedOn Specifies the date this resource was curated. pav:curatedBy gives the agents that performed the curation.

This property is normally used in a functional way, indicating the last curation date, although PAV does not formally restrict this.

pav:contributedBy Specifies an agent that provided any sort of help in conceiving the work that is expressed by the digital artifact.

Contributions can take many forms, of which PAV define the subproperties pav:authoredBy and pav:curatedBy; however other specific roles could also be specified by pav:contributedBy or custom subproperties, such as illustrating, investigating or managing the underlying data source. Contributions can additionally be expressed in detail using prov:qualifiedAttribution and prov:hadRole.

Note that pav:contributedBy identifies only agents that contributed to the work, knowledge or intellectual property, and not agents that made the digital artifact or representation (pav:createdBy), thus the considerations for software agents is similar to for pav:authoredBy and pav:curatedBy above.

pav:contributedBy is more specific than its superproperty dct:contributor - which might or might not be interpreted to also cover contributions to making the representation of the artifact.

pav:contributedOn Indicates the date this resource was contributed on. pav:contributedBy specifies the agents that contributed. This term is a superproperty of pav:authoredOn and pav:curatedOn, but can also be used for other kinds of contributions, such as illustrating or investigating. 
providedBy (see next subsection) gives a shortcut to express the publisher of the original work.

Figure 2 is an example illustrating the usage of PAV authoring terms. The figure depicts a partial representation of a hypothesis taken from the AlzSWAN knowledge base and published by the AlzSWAN team on behalf of AlzForum. Such a claim has been derived from a scientific publication and therefore is recorded as authored by the publication author. A curator performed on a particular date the task of encoding the research statements into the SWAN format.

\section{Provenance}

To encode provenance information specifying creation, retrieval, import and source access, PAV provides the properties presented in Table 2 .

While authoring terms like pav:authoredBy and pav: contributedBy describe who brought the underlying knowledge to light, the provenance term pav:createdBy describes who created the digital resource. For instance, in Figure 2, the creator is the user who formally encodes the claim, while the author is the person who wrote the original published article. In PAV, the digital artifact was pav:createdOn a given date, and it was pav:createdWith a specific software application. Similarly, a digitization of Charles Darwin's Galápagos notebook could be pav:createdOn 2006-10-06 T09:49:12Z and pav:authoredOn 183503-06 T00:00:00Z. (Note that this $x s d:$ dateTime string uses the convention of a zero timestamp as the exact time of day is unknown).
In PAV, we also distinguish between retrieving a resource 'as is' (pav:retrievedFrom), such as caching or downloading; importing a resource through a data transformation (pav:importedFrom) in order to fit it into an existing model, e.g., when converting a CSV file to an Excel spreadsheet; and accessing a resource (pav:sourceAccessedAt). The latter is useful when resources such as webpages are accessed but not cached or imported into the system.

As well as the above properties, PAV allows us to specify the agent that performed the task - pav:retrievedBy, pav: importedBy, pav:sourceAccessedBy - and the time when the task was performed - pav:retrievedOn, pav:importedOn and pav:sourceAccessedOn. For example, Figure 3 is a snippet specifying the record of a protein generated by importing data from the EntrezGene database [18]. The relationship pav:importedFrom is used to assert that the record is the result of a transformation process. In this specific case, an XML file has been translated into RDF according to a specific model (for example the lses namespace from the SWAN Ontology). The transformation is attributed to the agent that performed it - in this particular case a software agent - through the relationship pav:importedBy.

Additional properties can be used to enrich the provenance data. For instance pav:createdAt provides the geolocation of the agent when the artifact has been created.

\section{Versioning and evolution}

To avoid complexity, PAV adopts a 'snapshot'-based approach as opposed to a detailed process-oriented approach.

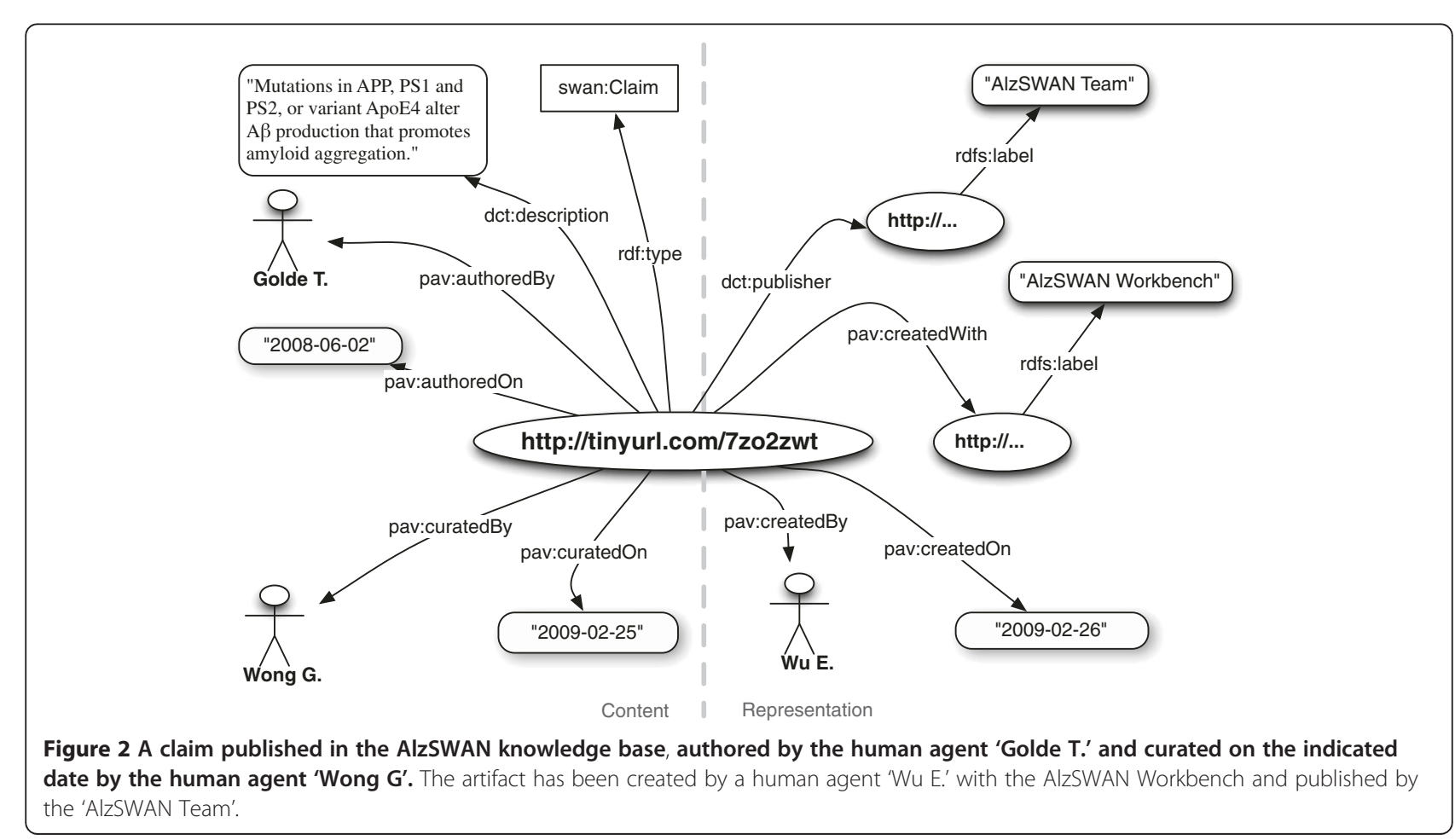




\section{Table 2 PAV provenance properties}

pav:createdBy

pav:createdOn

pav:createdWith

pav:createdAt

pav:retrievedFrom

pav:retrievedOn

pav:importedFrom

pav:importedBy

pav:importedOn

pav:lastRefreshedOn

pav:providedBy

pav:sourceAccessedAt
pav:retrievedBy

An agent primarily responsible for encoding the digital artifact or resource representation. This creation is distinct from forming the content, which is indicated with pav:contributedBy or its subproperties.

pav:createdBy is more specific than its superproperty dct:creator - which might or might not be interpreted to also cover the creation of the content of the artifact.

For instance, the author wrote' this species has bigger wings than normal' in his log book. The curator, going through the log book and identifying important knowledge, formalizes this as 'locus perculus has wingspan $>0.5 \mathrm{~m}$ '. The artifact creator enters this knowledge as a digital resource in the knowledge system, thus creating the digital artifact (say as JSON, RDF, XML or HTML).

A different example is a news article. pav:authoredBy indicates the journalist who wrote the article. pav:contributedBy can indicate the artist who added an illustration. pav:curatedBy can indicate the editor who made the article conform to the news paper's language style. pav:createdBy can indicate who put the article on the web site.

The software tool used by the creator to make the digital resource (say Protege, Wordpress or OpenOffice) can be indicated with pav:createdWith.

The date of creation of the digital artifact or resource representation. The agents responsible can be indicated with pav: createdBy.

This property is normally used in a functional way, indicating the time of creation, although PAV does not formally restrict this. pav:lastUpdateOn can be used to indicate minor updates that did not affect the creating date.

The software/tool used by the creator (pav:createdBy) when making the digital resource, for instance a word processor or an annotation tool. A more independent software agent that creates the resource without direct interactions by a human creator should instead be indicated using pav:createdBy.

The geo-location of the agents when creating the resource (pav:createdBy). For instance, a photographer takes a picture of the Eiffel Tower while standing in front of it.

The URI where a resource has been retrieved from. Retrieval indicates that this resource has the same representation as the original resource. If the resource has been somewhat transformed, pav:importedFrom should be used instead. This property is normally used in a functional way, although PAV does not formally restrict this.

An entity responsible for retrieving the data from an external source. The retrieving agent is usually a software entity, which has done the retrieval from the original source without performing any transcription.

Retrieval indicates that this resource has the same representation as the original resource. If the resource has been somewhat transformed, use pav:importedFrom instead.

The date the source for this resource was retrieved. This property is normally used in a functional way, although PAV does not formally restrict this.

The original source of imported information. Import means that the content has been preserved, but transcribed somehow, for instance to fit a different representation model by converting formats. The imported resource does not have to be complete but should be consistent with the knowledge conveyed by the original resource.

An agent responsible for importing data from a source given by pav:importedFrom. The importer is usually a software agent which has done the transcription from the original source. Note that pav:importedBy may overlap with pav:createdWith.

The date the resource was imported from a source given by pav:importedFrom. This property is normally used in a functional way, indicating the first import date, although PAV does not formally restrict this.

This property is normally used in a functional way, although PAV does not formally restrict this. If the resource is later reimported, this should instead be indicated with pav:lastRefreshedOn.

The date of the last import of the resource. This property is used if this version has been updated due to a re-import, rather than the import creating new resources related using pav:previousVersion.

The original provider of the encoded information (e.g. PubMed, UniProt, Science Commons).

The provider might not coincide with the dct:publisher, which would describe the current publisher of the resource. For instance if the resource was retrieved, imported or derived from a source, that source was published by the original provider. pav:providedBy provides a shortcut to indicate that original provider on the new resource.

A source which was accessed or consulted (but not retrieved, imported or derived from). For instance, a curator (pav: curatedBy) might have consulted figures in a published paper to confirm that a dataset was correctly pav:importedFrom the paper's supplementary CSV file.

Another example: I can access the page for tomorrow weather in Boston (http://www.weather.com/weather/tomorrow/ Boston+MA+02143) and I can blog 'tomorrow is going to be nice'. The source does not make any claims about the nice weather, that is my interpretation; therefore the blog post has pav:sourceAccessedAt the weather page.

pav:sourceAccessedBy The agent who accessed the source given by pav:sourceAcccessedAt . 


\section{Table 2 PAV provenance properties (Continued)}

\begin{tabular}{ll}
\hline $\begin{array}{l}\text { pav: } \\
\text { sourceAccessedOn }\end{array}$ & The date when the original source given by pav:sourceAccessedAt was accessed to create the resource. \\
& For instance, if the source accessed described the weather forecast for the next day, the time of source access can be crucial \\
& information. \\
& This property is normally used in a functional way, although PAV does not formally restrict this. If the source is subsequently \\
& checked again (say to verify validity), this should be indicated with pav:sourceLastAccessedOn. \\
& The date when the original source given by pav:sourceAccessedAt was last accessed and verified, especially when the source \\
pav: & has previously been pav:sourceAccessedOn when creating the resource. This property is normally used in a functional way, \\
sourceLastAccessedOn & although PAV does not formally restrict this. \\
& This property can be useful together with pav:lastRefreshedOn or pav:lastUpdateOn, but could also be used alone, for instance \\
& when a source was verified and no further action was taken for the resource.
\end{tabular}

Snapshots are identified by a URI and the free text property pav:version, and several snapshots of the same resource are related using pav:previousVersion, linking a version of the resource with the previous one of the same lineage. We use pav:derivedFrom to indicate an artifact as a derivation of another, not necessarily of the same lineage. Table 3 presents and describes PAV versioning properties.

As an illustrative example, Figure 4 specifies that the Hypothesis $\mathrm{A} 1$ is first created and then updated into A1' by the same human agent. A1 and A1' are two representation of the same version of the same hypothesis at two different points in time. The version A2 of the same hypothesis is created by another human agent having the same access rights. From the digital artifact standpoint it is accurate to say that the second version (A2) was created by a different agent than the first one, even if both versions are of the same lineage. In PAV the relationship pav:createdBy does not have any content authorship connotation. Computing the differences between the two versions allows attributing the intellectual property to the authors/curators who originated it. It is also possible to branch the lineage of digital artifacts through the relationship pav:derivedFrom. The property pav:lastUpdateOn is used to date when the digital artifact was last updated, indicating minor changes that did not signify a change of version (and therefore a new resource), such as fixing a typographical error.

Dublin Core Terms provides the property dct:source, which is used to specify "A Reference to a resource from

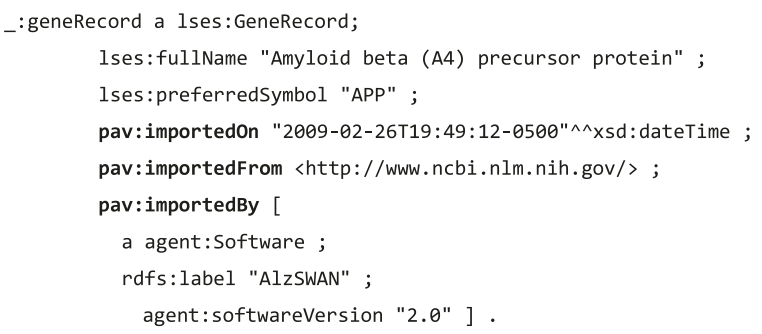

Figure 3 Example of import from the EntrezGene database expressed using Turtle notation [19]. The two namespaces /ses (life science entities [http://purl.org/swan/1.2/lses/] and agents [http://purl.org/swan/1.2/agents/] are part of the SWAN suite of ontologies. which the present resource is derived. The present resource may be derived from the Source resource in whole or part". Note, however, that this is more permissive, and therefore less specific, than pav:derivedFrom as it encompasses also format conversions.

\section{Multiplicity}

The properties defined by PAV do not have any multiplicity constraints. That means it is valid, for instance, to specify multiple authors using pav:authoredBy, or multiple contribution dates using pav:contributedOn. Some of the properties, like pav:retrievedFrom, pav:lastRefreshedOn or pav: version should still primarily be used in a functional way (as indicated in Tables 1, 2 and 3), as their interpretation could be difficult with multiple values. However PAV does not formally add functionality constraints to the OWL ontology.

Combinations of multiple agents, sources and dates may be used with PAV, but would mean that finer details are not fully preserved, such as who accessed which source when. This is the result of a compromise between simplicity and completeness when designing PAV. It is recommended that applications that want to keep those details also provide an accompanying PROV-O trace, as exemplified in Figure 5.

In this example PAV will tell us there are 3 authors, and but the resource has only got a single authoring date (the last time of authoring). We do not know from PAV alone when the different authors participated, but the expanded PROV-O trace, which qualifies the implied prov:wasAttributedTo relations, can detail those dates using prov:atTime. Here the nature of the individual attributions are also indicated using prov:hadRole and a custom role vocabulary ex:, and the prov:atLocation indicates that :khalid and :stian were in the same office, authoring at the same time.

In some cases it might not be appropriate to attribute the individual agents directly, for instance a report authored by a committee where the individual members have discussed and voted over the content. We recommend for such cases to be represented as a single identified agent, typically a prov:Organization, and the individual members represented using the Collection Ontology [20], as shown in Figure 6. 


\section{Table 3 PAV versioning properties}

pav:version

pav:

previousVersion

lastUpdateOn

The version identifier of a resource. This is a free text string, typical values are ' $1.5^{\prime}$ ' or ' 21 '. The URI identifying the previous version can be provided using pav:previousVersion. This property is normally used in a functional way, although PAV does not formally restrict this.

The previous version of a resource in a lineage. For instance a news article updated to correct factual information would point to the previous version of the article with pav:previousVersion. If, however, the content has significantly changed so that the two resources no longer share lineage (say a new article that talks about the same facts) they can instead be related using pav: derivedFrom.

This property is normally used in a functional way, although PAV does not formally restrict this. A version identifier for a resource can be provided using the data property pav:version.

pav:derivedFrom Derived from a different resource. Derivation concerns itself with derived knowledge. If this resource has the same content as the other resource, but has simply been transcribed to fit a different model (like XML to RDF or SQL to CSV), use pav:importedFrom. If the content has been further refined or modified, use pav:derivedFrom.

Details about who performed the derivation (e.g. who did the refining or modifications) may be indicated with pav:contributedBy and its subproperties.

The date of the last update of the resource. An update is a change which did not warrant making a new resource related using pav:previousVersion, for instance correcting a spelling mistake. This property is normally used in a functional way, although PAV does not formally restrict this.

\section{Contribution roles}

While PAV properties like pav:retrievedBy and pav:createdWith are fairly specific, yet generally applicable; different domains will vary in their understanding of what in PAV would constitute the roles of pav:authoredBy, pav:curatedBy or pav:contributedBy. For instance, the International Workshop on Contributorship and Scholarly Attribution 2012 [21] presented a survey where "authorship" was found to regularly include roles such as design of experimental methods and statistical analysis, but also software development, preparing graphics and managing a laboratory. The presented text analysis on acknowledgement sections in academic papers identified non-author contributions like funding, technical assistance, data contribution, and animal assistance.

It is out of scope for PAV to try to model this wide range of contributorships, but we do note that ontologies such as SPAR's Publishing Roles Ontology [22] define roles like pro: illustrator, pro:critic and pro:editor which would be appropriate to use with prov:hadRole in the pattern shown in Figure 5. Additionally, third-party subproperties, e.g. of pav: contributedBy and pav:authoredBy, can be created to further specify the form of the contribution, utilizing PAV as a common platform for attributions across domains.

\section{Who is using PAV}

PAV has successfully been applied by several projects in academia and in industry due to it being compact and easy to understand. Besides the SWAN project, for which PAV was originally developed, it has been used in the Annotation Ontology (AO) [23]; the Domeo Annotation Tool [24]; the Nanopublications specification [25]; the Open PHACTS dataset description specification [26-28]; the Wf4Ever Research Objects [29]; and the Elsevier Satellite article annotation format [30]. For its most recent release, we updated PAV to include a mapping to the PROV-O ontology [3],

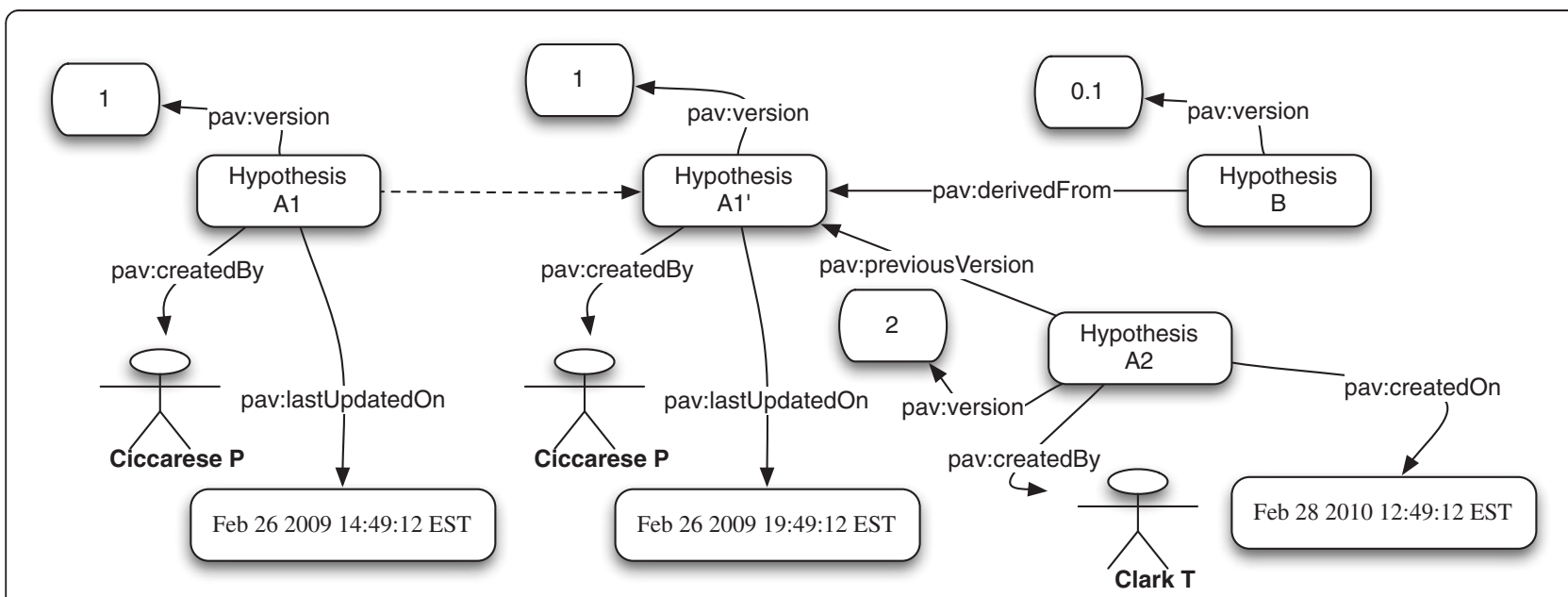

Figure 4 Example illustrating versioning with PAV. Hypothesis A1 and Hypothesis A1' have the same URI, they are representing the same resource at different points in time. 


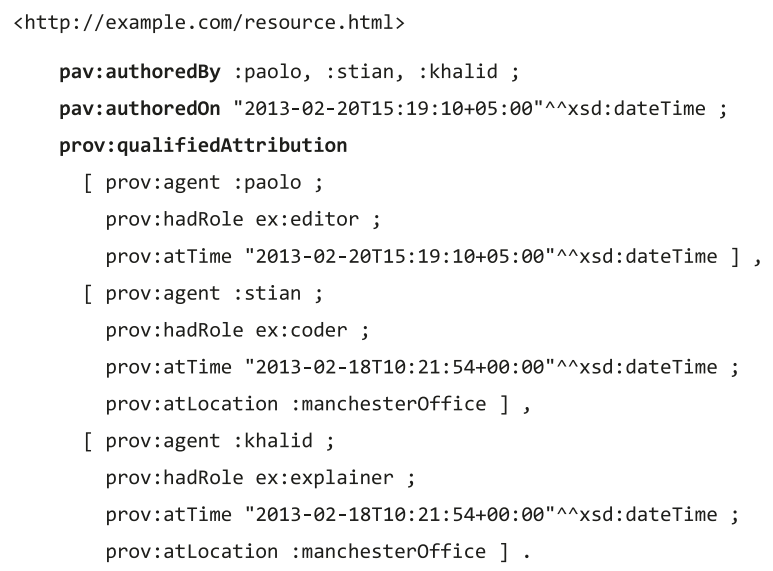

Figure 5 Example illustrating how PROV-O can be combined with PAV in order to provide a more detailed provenance record.

enabling a level of compatibility for all PROV-based tools for analyzing and combining provenance. Figure 7 illustrates PAV's relation to these projects.

\section{Annotation ontology and Domeo annotation tool}

PAV is used extensively by many of the applications making use of the Annotation Ontology (AO) [23] for anchoring annotations to online resources. One such application is the Domeo Web Annotation Toolkit [24], a collection of software components that provides a rich set of features including:

- semantically annotating online HTML and XML documents;

- sharing the annotation in RDF; and

- searching the annotation while leveraging semantic inference.

Domeo, as well as the other AO applications, provide a constant stream of requirements and feedback for testing and improving the PAV model.

In Figure 8 we represent a common scenario where the annotation artifact is digitizing an annotation that has been originally performed on the physical manifestation of a picture. In other words: Khalid scribbled a note on a classic printed picture; Paolo found a digital version of that picture and interpreted the handwritten note by Khalid and passed it along to Stian who, using Domeo, created an AO artifact representing the whole scenario. Khalid is the author (pav: authoredBy) of the original note, Paolo is the curator (pav:
curatedBy) of that content and Stian is the creator (pav:createdBy) of the digital artifact.

\section{Open PHACTS dataset descriptions}

The aim of the Open PHACTS project $[26,27]$ is to facilitate improvements in drug discovery using semantic web standards and technologies. Drug discovery requires the integration of data from many data sources covering chemical compounds (e.g. ChemSpider [31] and ChEMBL [32]), proteins (e.g. UniProt [11,12]), and drug interactions (e.g. DrugBank [33]). As such, the project requires accurate descriptions of the datasets they have used, identifying the particular versions, so that data provenance can be returned to the users.

In the specification [28], Open PHACTS recommend the use of existing vocabularies: Vocabulary of Interlinked Datasets (VoID) [34]; Dublin Core Terms (DC Terms) [2]; Friend of a Friend (FOAF) [35]; and PAV. VoID itself does not define any new provenance terms, but specifies a pattern of using DC Terms properties for purposes of recording provenance, such as dct:creator, dct:contributor and dct:source [36].

The Open PHACTS dataset specification, which uses a specialization of VoID, also specifies patterns of recording provenance, but from the provenance-related terms of DC Terms, only uses dct:publisher and dct:issued, and primarily recommends the PAV properties: pav:version, pav: previousVersion, pav:retrievedFrom, pav:importedFrom, pav: importedOn, pav:importedBy, pav:derivedFrom, pav:createdOn, pav:createdBy, pav:createdWith, pav:authoredBy, pav: authoredOn, pav:lastRefreshedOn and pav:lastUpdateOn.

The Open PHACTS VoID editor [37], shown in Figure 9, provides a wizard-like web interface for generating dataset descriptions, including the above-mentioned PAV properties.

\section{Nanopublications}

Nanopublications [38] have been proposed by the ConceptWeb Alliance and the Open PHACTS project as a new means for publishing and citing specific core scientific statements. A nanopublication is composed of two basic elements: an assertion, and the provenance of that assertion. The former is used to express a single scientific fact, whereas the second is used to provide supporting evidence, in addition to the nanopublication attribution, i.e. its authors and other metadata information. To express provenance information, the Nanopublication specification [25] recommend the use of PAV and Open Provenance Model (OPM) [4]. For instances, the examples of nanopublications advertised on the

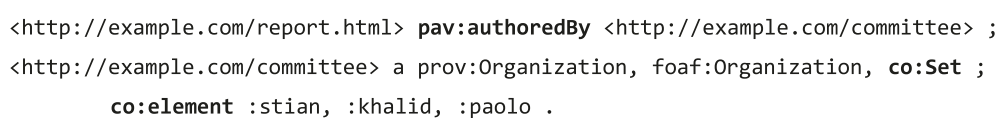

Figure 6 Example illustrating how Collection Ontology (CO) can be used with PAV in order to encode a collection (set) of people. 


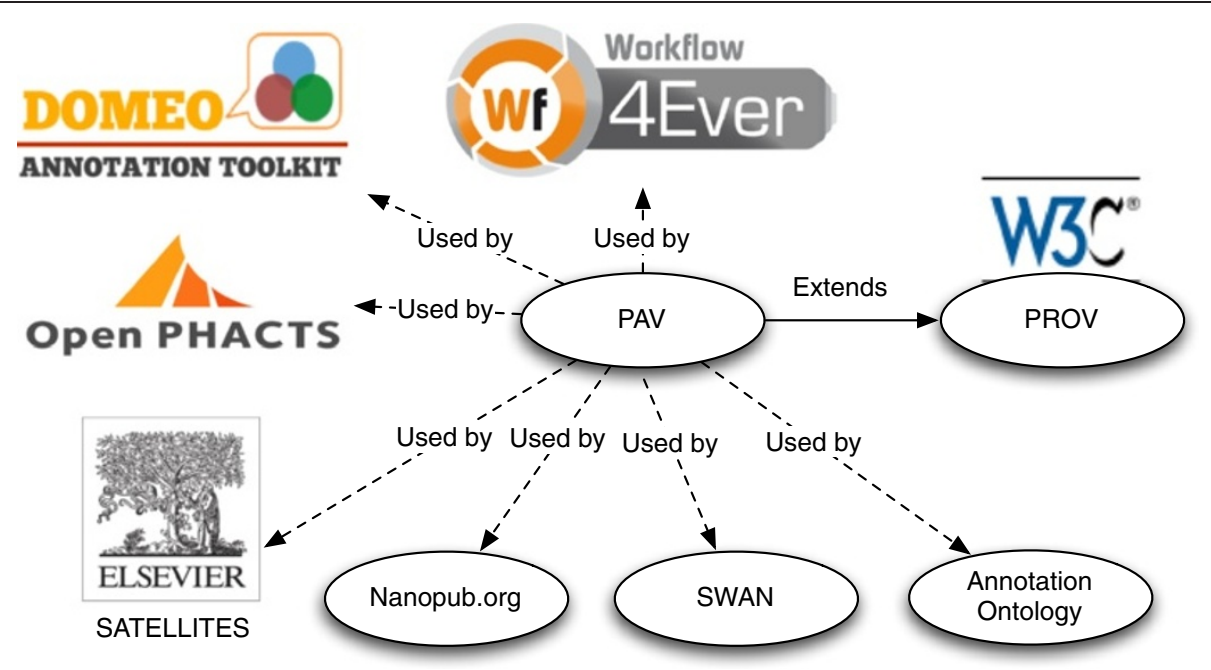

Figure 7 Relationships between the PAV ontology, the PROV ontology and all the projects listed in this article making use of PAV.

nanopublication official website [39] make use of the following PAV properties: pav:authoredBy, pav:createdBy, pav:version.

To illustrate this use of PAV, the RDF snippets in Figures 10, 11 and 12 specify a nanopublication assertion and its provenance. These snippets are taken from a real world nanopublication [40], here slightly simplified for brevity. Notice that the attribution information (Figure 12) uses pav:authoredBy and pav:createdBy to distinguish the author (the scientist making the claim) from the artifact creator (who formed the nanopublication as RDF). The nanopublication itself is given a version number with pav:version.

Note that the process of structuring a nanopublication could also be considered a kind of curation, as in order to serialize the RDF the creator has to also look up identifiers for genes and symptoms, a content refinement as

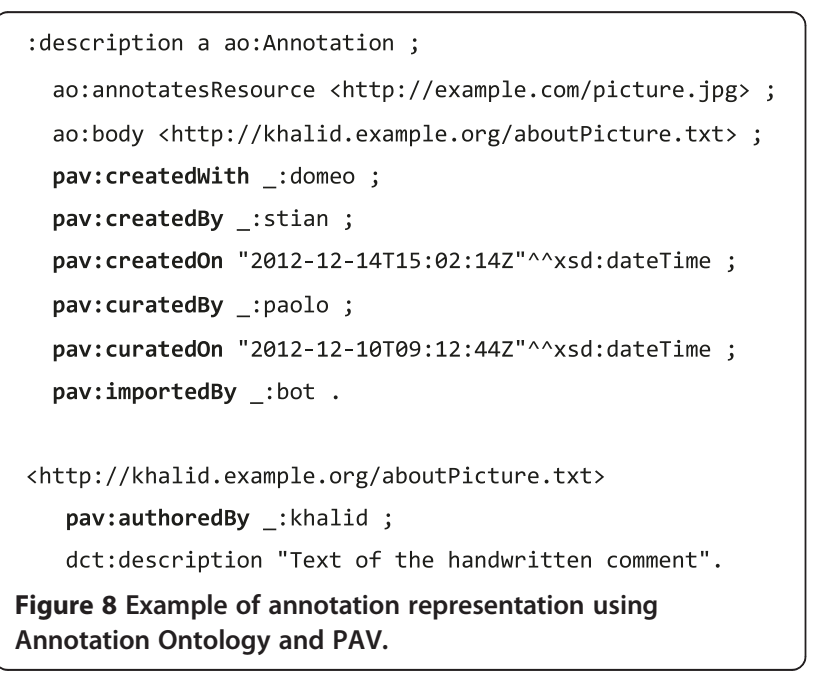

such identifiers presumably where not in the original content. The nanopublication we found as an example did however not specify pav:curatedBy, so from the above we can't tell anything more about the curation aspect of the nanopublication.

\section{Elsevier satellite}

PAV has been used, in conjunction with DC Terms, as the provenance ontology in the Elsevier Satellite annotation format [30]. The Satellite format is a linked data compliant data format to capture, store and expose metadata objects using open standards based metadata frameworks e.g. SKOS [43], DCMI and SWAN. Satellite format uses PAV 1.2 as originally specified in the SWAN suite of ontologies [9].

In Satellite format, PAV differentiates the provenance properties used for the metadata container and those used for the contained metadata items. Satellite uses the dct:date and dct:creator predicates to provide information on the item being described by the metadata. Satellite uses the pav:createdOn and pav:createdBy predicates in a header to describe the origin of the Satellite metadata itself.

\section{Research objects}

The notion of "Research Object" [29] was coined by the Wf4Ever project as an abstraction for the management of containers of sets of objects. In Wf4Ever, research objects bundle investigation-related resources such as documents, presentations, workflows, datasets, etc. Research Objects, their constituent resources, and their relationships, are described and annotated using existing vocabularies, including PAV. Specifically, the Wf4Ever Research Object Model [44] uses PAV to: 


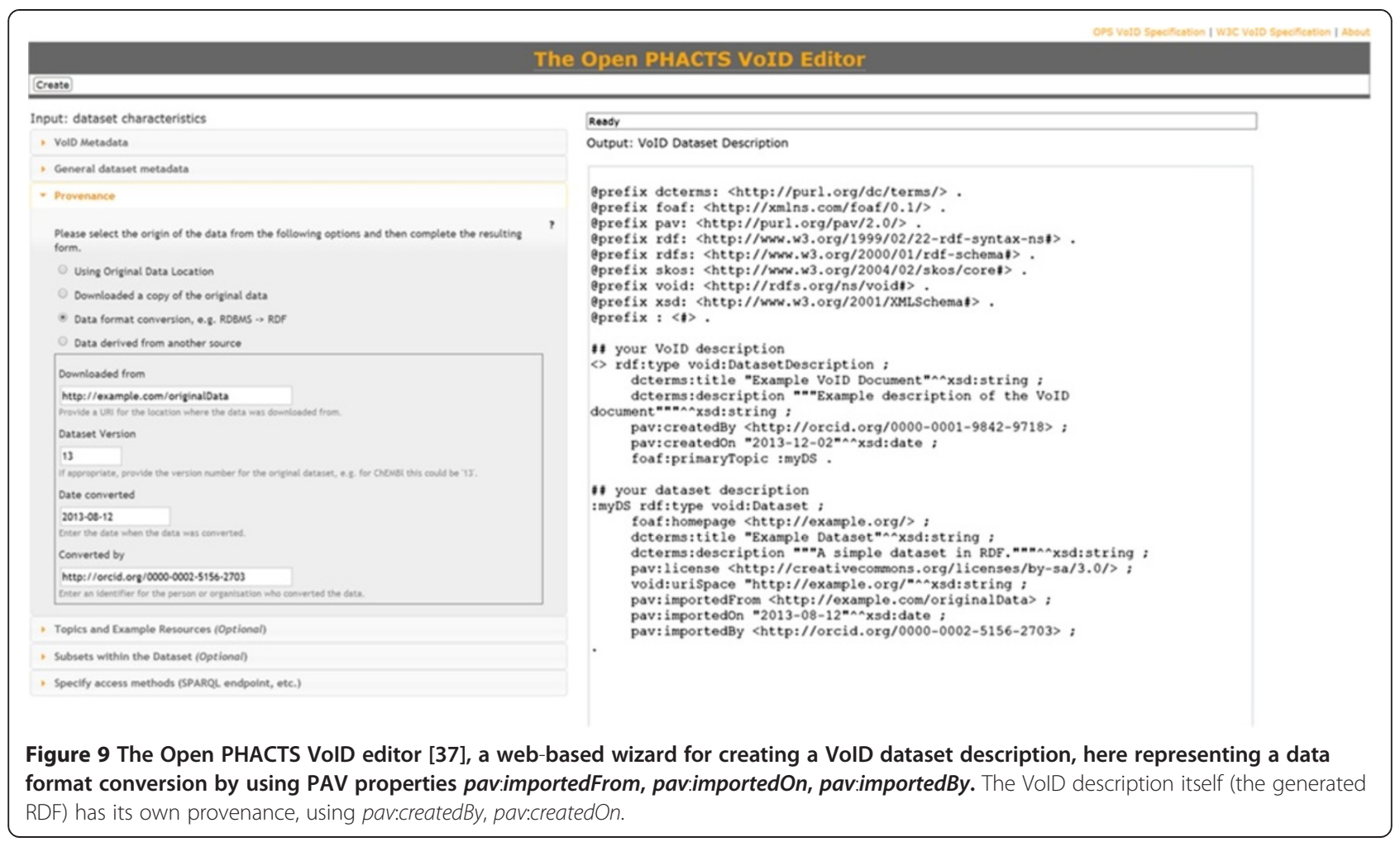

- Identify and distinguish the people ("Agents") who contributed to a Research Object (and its constituent resources) from those who created them. For instance, a hypothesis document could have been authored by a PhD student, but uploaded to a research object by their supervisor (thus creating the digital resource)

- State the time at which a Research Object, or a resource thereof, was last updated.

- Track the versions and origin of replicated resources, such as recording provenance for resources and user annotations which have been imported from and retrieved from third-party repositories using automated tools.

\section{PROV-O and PAV mapping}

The earlier version 1.2 of PAV was recognized (as a component of the SWAN ontology) by the W3C Provenance Incubator Group [45] and was one of the foundational models [46] on which the requirements for the general provenance model later defined by the W3C Provenance Working Group.

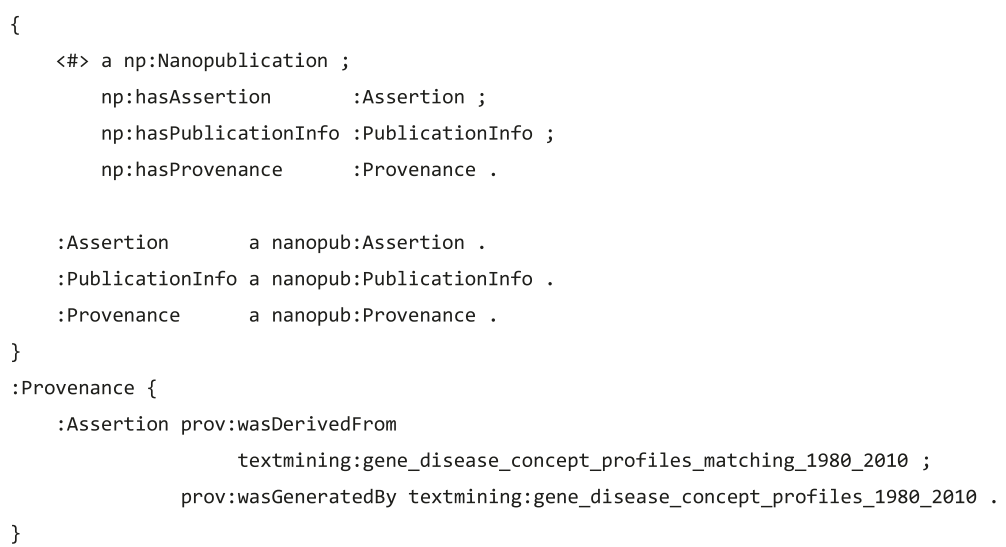

Figure 10 Gene disease nanopublication example, in TriG format, adapted from [40]. The nanopublication is expressed as three named graphs: the Assertion which expresses the claim of this nanopublication (Figure 11), the Publication/nfo, which details the attributions of this assertion (Figure 12), and Provenance, relating this nanopublication to the original data it was derived from (shown above). 


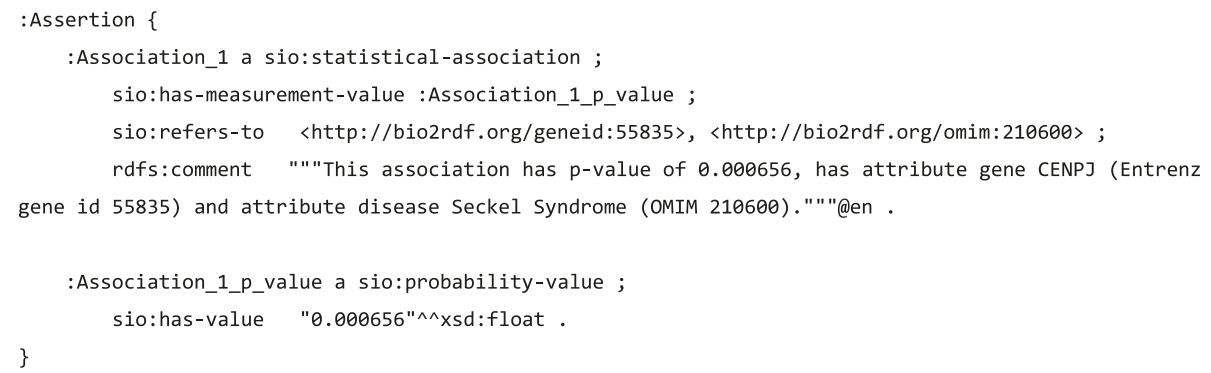

The W3C Provenance working group later released the PROV-O Ontology [3] as a Recommendation. PROV-O provides a general way to describe provenance relations using OWL. It describes provenance as a set of interactions between Entities, Agents and Activities. Interactions can be described using direct relations like prov:wasGeneratedBy; or they may be described with qualified indirect relationships, using classes like prov: Generation. The latter allows assignment of roles to agent participations and other details such as timestamp and location.

PROV-O is a generic framework for describing provenance in a whole range of applications, but used alone it lacks necessary detail for the more specific provenance of authoring and versioning that arise from our use cases. PAV can be a useful specialization of PROV-O by providing simple relationships for expressing common provenance for digital artifacts. Therefore PAV, starting from version 2.1, introduced a mapping from PAV to PROV-O using subproperties, as detailed in Table 4:

In PROV-O, entities are considered immutable, and different states for the purposes of provenance are represented as different entities, each with their own provenance. The W3C Provenance Working Group has published a note "Dublin Core to PROV Mapping" [47], proposing a subproperty mapping from Dublin Core
Terms to PROV-O, in addition to a "complex mapping" by using SPARQL CONSTRUCT to create detailed PROV-O traces. This note highlights the difference between Dublin Core and PROV-O resources: while the former conflates more than one version or "state" of the resource in a single entity, the latter proposes to separate all of them.

We have provided a subproperty mapping from PAV to PROV-O, which implies a similar entity conflation, by attaching all properties to the same resource rather than introducing intermediate entities, which would be required to give a detailed PROV-O trace of the activities that lead to the generation of the final resource state. The combination of OWL/RDFS reasoning and the PROV inference rules [48] means we can infer further PROV statements such as entity generation and activity association from a single PAV statement, shown in Figure 13.

Note that some information is not preserved in these inferred statements, e.g. the distinction between authors and curators; and the PAV statements of authorship and authorship time are detangled into separate existential variables and PROV-O statements.

A more integrated mapping from PAV properties to such chains of PROV-O activities and entities could be formulated in a similar fashion to that shown in [47],

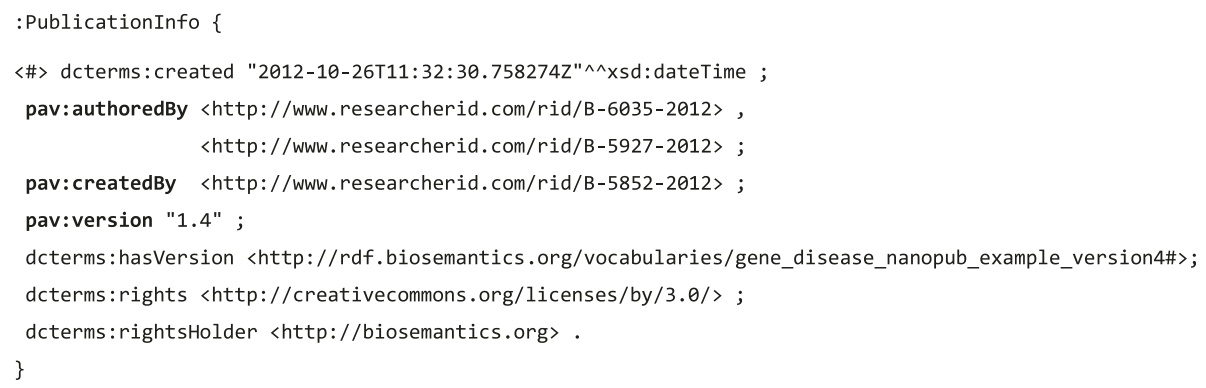

Figure 12 Nanopublication attribution specifying attribution information of the nanopublication in Figures 10 and 11 . PAV is used to distinguish between the author of the nanopublication (the scientists who made the assertion expressed in Figure 10), and the creator of its digital representation, who in this case expressed the assertion as an RDF graph. The nanopublication is given a pav:version, also identified using dcterms:hasVersion. (The original RDF uses the PAV 1.2 term "versionNumber" which was renamed to "version" in PAV 2.0.). 
Table 4 Mapping from PAV to PROV-O

\begin{tabular}{|c|c|c|}
\hline PROV-O superproperty & PAV property & Rationale \\
\hline \multirow[t]{7}{*}{ prov:wasAttributedTo } & pav:createdBy & The creator agent participated in some activity that generated the entity. \\
\hline & pav:createdWith & The software agent participated in some activity that generated the entity. \\
\hline & $\begin{array}{l}\text { pav: } \\
\text { contributedBy }\end{array}$ & The contributor participated in some activity that generated the entity. \\
\hline & pav:authoredBy & The author participated in some activity that generated the entity. \\
\hline & pav:curatedBy & The curator participated in some activity that generated the entity. \\
\hline & pav:importedBy & $\begin{array}{l}\text { The agent (usually software in this case) participated in some import activity, which generated the } \\
\text { entity. }\end{array}$ \\
\hline & pav:retrievedBy & $\begin{array}{l}\text { The agent (usually software in this case) participated in some retrieval activity, which generated the } \\
\text { entity. }\end{array}$ \\
\hline \multirow[t]{2}{*}{$\begin{array}{l}\text { prov:wasDerivedFrom \& } \\
\text { prov:alternateOf }\end{array}$} & $\begin{array}{l}\text { pav: } \\
\text { importedFrom }\end{array}$ & $\begin{array}{l}\text { Import is a transformation of an entity into another. As the resulting entity is presenting aspects of } \\
\text { the same thing, it is also an prov:alternateOf the original. }\end{array}$ \\
\hline & $\begin{array}{l}\text { pav: } \\
\text { retrievedFrom }\end{array}$ & $\begin{array}{l}\text { Retrieval is construction of an entity into another. As the resulting entity is essentially (bytewise) the } \\
\text { same, i.e. presenting aspects of the same thing, it is also an prov:alternateOf the original. Some aspects } \\
\text { of the original entity (like its dct:publisher) might not be shared, and therefore prov:specializationOf is } \\
\text { not an appropriate superproperty. }\end{array}$ \\
\hline prov:wasDerivedFrom & pav:derivedFrom & $\begin{array}{l}\text { Derivation is an update of an entity, resulting of a new one. Note that pav:derivedFrom is more } \\
\text { specific than prov:wasDerivedFrom, and does not cover "minor" derivations as with pavimportedFrom } \\
\text { and pav:retrievedFrom. PAV derivation implies that additional knowledge has been contributed, } \\
\text { curated or authored. }\end{array}$ \\
\hline prov:wasRevisionOf & $\begin{array}{l}\text { pav: } \\
\text { previousVersion }\end{array}$ & $\begin{array}{l}\text { The new version is a revised version of the original. pav:previousVersion is more specific than prov: } \\
\text { wasRevisionOf because it is intended for minor updates and corrections, and typically would be used } \\
\text { with linearly incremental pav:version numbers. Significant changes (contributing new knowledge) } \\
\text { should be indicated with pav:derivedFrom. }\end{array}$ \\
\hline prov:wasInfluencedBy & $\begin{array}{l}\text { pav: } \\
\text { sourceAccessedAt }\end{array}$ & $\begin{array}{l}\text { The source Entity has an effect on the character, or development of the entity. The PAV term is a weak } \\
\text { indication that another resource was consulted (for instance as part of curation), but without the } \\
\text { new entity being directly derived from the source. The prov:hadPrimarySource is not an appropriate } \\
\text { superproperty, as it implies a stronger statement, giving the source the status of a primary source } \\
\text { and derivation. As the resource is not necessarily based on or transformed from the consulted } \\
\text { source, we can't assume prov:wasDerivedFrom as a superproperty. }\end{array}$ \\
\hline
\end{tabular}

which explores complex mappings of Dublin Core Terms to detailed PROV-O patterns. For instance, unrolling PAV import statements to PROV-O activities could create triples as shown in Figure 14.

Detailing such a mapping is currently work in progress, and would have to balance logical correctness vs. usefulness, for instance the above assumes that all PAV import statements describe the same activity, but if there are multiple pav:importedFrom statements and multiple pav:importedBy statements we cannot be certain about the extent of that import activity.
The authors believe that the current PROV-O subproperty mapping is liberal enough to allow PAV to complement more detailed provenance traces using PROV-O, while enabling inferences to compatible PROV-O statements.

In order to demonstrate PAV interoperability with PROV-O, we wanted to make use of the PROV-O mapping, so that PROV-O statements could be inferred from PAV statements using a standard OWL reasoner. We then wanted to test if a PROV-O consuming tool was able to understand the statements.

$$
\text { <http://example.com/resource.html> pav:authoredBy :paolo . }
$$

$$
\begin{aligned}
\text { (subproperty) } \rightarrow & \langle\text { http://example.com/resource.html> prov:wasAttributedTo : paolo . } \\
\text { (attribution-inference) } \rightarrow & \langle\text { http://example.com/resource.html> prov:wasGeneratedBy _:activity . } \\
& \text { :paolo prov:wasAssociatedWith _:activity . }
\end{aligned}
$$

Figure 13 Inferences from PAV authorship to existential PROV-O activities. pav:authoredBy is subproperty of prov:wasAttributedBy, which, according to PROV constraint attribution-inference imply that there existed some _:activity that generated the resource and which :paolo was associated with. 


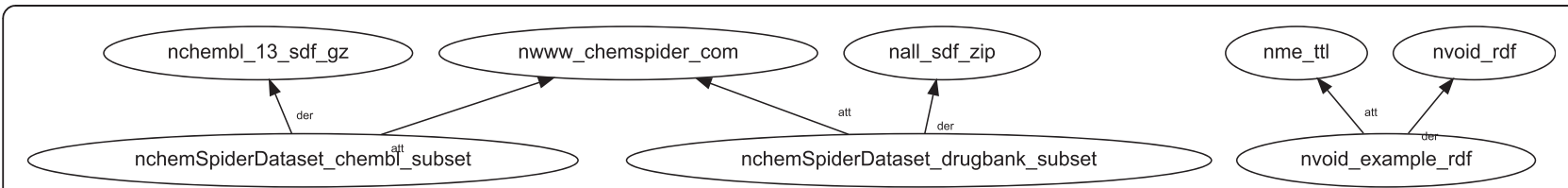

Figure 14 Visualization of ChemSpider VoID provenance. Both subsets are attributed to chemspider.com (pav:retrievedBy), and derived from gz/zip files (pav:retrievedFrom). The VolD file itself is attributed to "me" (pav:createdBy) and derived from void.rdf (pav:derivedFrom). Note that the labels above are generated by ProvToolbox based on the URIs - the n-prefix indicates a prov:Entity. Figure converted from SVG diagram which was produced by Taverna workflow [46].

To perform this experiment, we used Taverna Workbench v2.4 [49] to build a workflow [50] using the OWL reasoner Pellet v2.3.0 [51], and then visualized the inferred statements as a diagram (SVG) by using ProvToolbox v0.1.2 [52], which understands PROV-O. We executed this workflow against the VoID example [53] in the Open PHACTS dataset specification [28] to visualize its PAV statements. The generated provenance diagram is shown in Figure 14.

\section{Method}

The PAV ontology was developed with the aim of enabling traceability of scientific results and their representations. The design was driven by real requirements, which initially stemmed from the AlzSWAN project, and later were expanded to requirements from other projects. DC terms and Open Provenance Model [4] were available at that time, and could have been used. However, they were found not to be suitable. Specifically, DC Terms conflates different agent roles that the participants in the SWAN project want to distinguish, in particular, authorship, creation and contribution. OPM adopts a process-oriented view of lineage by detailing the processes whereby artifacts are used and generated, and the agents that controlled those processes. Instead, the requirements elicited in the context of the SWAN project, and other projects later on, targeted mainly the expression of lineage in terms of the relationships between the artifacts, and the relationships between the artifacts and the agents involved in their creation, authorship or curation.

For these reasons, we decided to develop a new vocabulary that specifically addressed the SWAN project requirements. In doing so, the following principles were followed:

Keep the ontology lightweight: Experience suggests that a complex and large vocabulary (albeit well crafted) is likely not to be adopted by users. Therefore, the authors of PAV were keen to have a minimal set of terms (properties) that cater for the needs identified in the context of the AlzSWAN project. In particular, PAV does not attempt to model the complete chain of process-oriented provenance.

Favoring Incremental (and organic) development: Rather than trying to design an ontology and convince the users to utilize it, the development of the PAV ontology went through cycles in which the ontology designers communicated with end users and examined how the ontology is used in practice. Modifications and additions were then made based on the observations made in each cycle.

Reuse and recommend existing vocabularies when they cater for given requirements: For those requirements supported by existing vocabularies, the authors of the PAV ontology strived to either recommend their reuse or (when necessary) extend them. In this respect, we have shown in the previous section, how PAV extends terms from the PROV-O ontology.

The PAV ontology is currently in its second version. Since its inception, PAV has gained momentum, and it is now adopted by several vocabularies and projects. It is increasingly viewed as one of the main vocabularies for specifying provenance information in the biomedical semantics field. Some of the applications and projects that have adopted PAV are indicated in the Results section. In 2009, PAV was used as one of its starting vocabularies by the W3C Provenance Incubator group. The Incubator group preceded the W3C Provenance Working Group, which made the current PROV specifications.

\section{Discussion}

In this section, we analyze and compare three proposals we have found to be relevant to PAV: Dublin Core Terms, BIBFRAME and Provenance Vocabulary (PRV). We then close the article by presenting some concluding remarks.

\section{Dublin core terms}

The Dublin Core Terms vocabulary provide terms such as dct:contributor and its subproperty dct:creator, and we have argued that they conflate the roles of content authoring, knowledge curation and representation creation. Although our presented use-cases highlights the importance of distinguishing these in the setting of formal knowledge representation, the ambiguous definition of dct:creator also means that its value for stating consistent provenance is significantly reduced on the web in general. In cases where the content author and representation creator are different, common use of DC Terms 
[2] is often to attribute only one of these, but which one depends on the application. A use case could be a corporate blog where a webmaster (Bob) types in an announcement, which the CEO (Alice) sent in an email. Some blog platforms would automatically represent the currently logged in user (Bob) as the dct:creator, other platforms might allow the webmaster to select an author (Alice) from the corporate directory and would instead represent her as the dct:creator.

Using PAV, the blog platform can be more precise about the provenance of the post. When the platform has no user interface for describing the author, the safest would be to present pav:createdBy for the current user. If the user interface allows selecting a different author, then both pav:authoredBy and pav:createdBy can be supplied. Enterprise publishing platforms could also indicate curation (e.g. hyperlinks and textual formatting) with pav:curatedBy and additional contributions (such as adding an illustration) with pav:contributedBy.

Dublin Core Terms defines terms that may cover some provenance aspects (dct:isFormatOf, dct:source, dct:isVersionOf, dct:replaces), however DC Terms concerns itself primarily with catalogue metadata for a resource, while PAV has a bigger focus on entity-agentdriven provenance.

For instance, dct:isFormat $f$ is an existential statement that there is a different representation of the same content, while pav:importedFrom also implies directionality and a transformation step which was performed by an agent, indicated with pav:importedBy. The former term is useful for finding alternate representations, while the latter PAV relation gives lineage to the resource, which can be beneficial for instance to track down the source of an inconsistency or to verify that data is current.

As dct:creator can be seen to cover both content authoring and creating its representation, we have defined both pav:authoredBy and pav:createdBy to be subproperties of dct:creator, while its superproperty dct: contributor is a superproperty of pav:contributedBy; here the PAV term only covers contributions to the work or content, while dct:contributor may also cover representational contributions such as scaling an image or converting HTML to PDF.

Other PAV properties have not been mapped to DC Terms in the OWL ontology. Part of the reason for this is that the DC Terms vocabulary is not fully OWL compatible (e.g. properties are not declared as either annotation or object properties), another is that we found the more bibliographic DC Terms to be hard to align with the provenance oriented aspect of PAV using strict OWL property hierarchies.

In order to clarify the differences between the remaining properties that might seem similar between PAV and Dublin Core Terms and to relate the two vocabularies in detail, we defined a SKOS mapping [54]. As the differences are often conceptual we found the use of SKOS [43] more beneficial than a formal OWL mapping. The most significant mappings are shown in Table 5 with their rationale:

\section{BIBFRAME}

The Library of Congress officially launched its Bibliographic Framework Transition Initiative (BIBFRAME) $[55,56]$ initiative in May 2011. The initiative aims to reenvision the current standard for bibliographic exchange (MARC 21) [57] and implement a new bibliographic environment for libraries that makes "the network" central and interconnectedness commonplace, using semantic web technologies. As PAV can be used to express attribution of both digital resources and traditional publications, and BIBFRAME is an emerging standard within the library community, we here explore BIBFRAME and compare it with PAV.

BIBFRAME revolves around two main concepts: the Creative Work; and the Instance, reflecting an individual, material embodiment of the Work. These are similar distinctions to the Work and Manifestation dichotomy in the original Functional Requirements for Bibliographic Records (FRBR) [58] model, or in other related models such as the FRBR-aligned Bibliographic Ontology (FaBiO) [59]. An example of the BIBFRAME twolevel model is depicted in Figure 15.

Although PAV itself does not distinguish between work and instances, the distinction between content and its representation is at the core of PAV; exemplified by pav:authoredBy vs. pav:createdBy. There is however nothing inherent with PAV itself that prevents its usage with separate Work and Instance resources. In fact, the PROV-O property prov:specialization $O f$ is intended for modeling abstraction levels, so if a $b f:$ Work is pav: authoredBy Alice, and a bf:Instance is a prov:specialization $O f$ the work, then the instance can be implied to also be pav:authoredBy Alice.

For bibliographic data, multiple abstractions levels such as in BIBFRAME and FRBR are elegant and useful, but for many other use cases, such as for provenance of a blog post or nanopublication, the separation of instance and work can be inconvenient, hard or even impossible to achieve. PAV, as a general vocabulary for provenance and authoring of resources, is applicable in both approaches.

\section{Provenance vocabulary}

While PAV allows expression of data sources (pav:sourceAccessedAt) and derivations (pav:derivedFrom, pav: importedFrom), the Provenance Vocabulary (PRV) $[5,61]$ is an extension of PROV-O to express more detailed provenance of data items on the web, by forming chains 
Table 5 SKOS mappings of applicable PAV terms to Dublin core terms

\begin{tabular}{|c|c|}
\hline SKOS mapping & Rationale \\
\hline $\begin{array}{l}\text { pav:authoredBy skos:broadMatch dct: } \\
\text { creator }\end{array}$ & $\begin{array}{l}\text { Broad match due to the common usage of dct:creator to mean the creator of the Work rather than just } \\
\text { the creator of the particular resource. Solely creating the representation of a resource is in PAV covered } \\
\text { instead by pav:createdBy, but would often also be covered by dct:creator, therefore this is not a skos: } \\
\text { closeMatch. }\end{array}$ \\
\hline $\begin{array}{l}\text { pav:contributedBy skos:closeMatch dct: } \\
\text { contributor }\end{array}$ & $\begin{array}{l}\text { Close match due its the common usage to mean someone who added to the Work of the resource } \\
\text { (usually not just the digital representation), but not skos:exactMatch as purely representational } \\
\text { contributions represented with dct:contributor should be mapped to pav:createdBy. }\end{array}$ \\
\hline $\begin{array}{l}\text { pav:createdBy skos:broadMatch dct: } \\
\text { creator }\end{array}$ & $\begin{array}{l}\text { A PAV creator is a particular kind of DC Terms creator, which made the digital representation of the } \\
\text { resource. }\end{array}$ \\
\hline $\begin{array}{l}\text { pav:importedFrom skos:broadMatch } \\
\text { dct:source }\end{array}$ & Imported is a specialization of being derived from the related resource in whole. \\
\hline $\begin{array}{l}\text { pav:importedFrom skos:broadMatch } \\
\text { dctisFormatOf }\end{array}$ & $\begin{array}{l}\text { The resulting resource is substantially the same as the source, but in another format. However imported also } \\
\text { implies provenance of a directed transformation from the original, at a given time and performed by an } \\
\text { agent, and hence this is a broad match. }\end{array}$ \\
\hline $\begin{array}{l}\text { pav:importedBy skos:broadMatch dct: } \\
\text { creator }\end{array}$ & $\begin{array}{l}\text { The agent importing is essentially a specialized creator of the new resource, hence has close match dct: } \\
\text { creator. In common use of DC Terms the extent of the transformation work might however affect whether } \\
\text { a dct:creator corresponds to an importer or author. }\end{array}$ \\
\hline $\begin{array}{l}\text { pav:derivedFrom skos:broadMatch dct: } \\
\text { source }\end{array}$ & $\begin{array}{l}\text { A related resource from which the described resource is derived, but pav:derivedFrom is more specific (skos: } \\
\text { broadMatch) than dct:source, as it requires further contributions to the content, and does not cover say } \\
\text { pav:importedFrom or pav:retrievedFrom. }\end{array}$ \\
\hline $\begin{array}{l}\text { pav:derivedFrom skos:narrowMatch } \\
\text { dctisVersionOf }\end{array}$ & $\begin{array}{l}\text { pav:derivedFrom do point to a resource of which the 'described resource is a version, edition, or adaptation', } \\
\text { but also allow further derivations, and so has a narrow match dct:isVersionOf. The pav:derivedFrom does } \\
\text { require such contributions to be in the form of content and not just representation, which corresponds } \\
\text { closely to dct:isFormatOf 's description' Changes in version imply substantive changes in content rather than } \\
\text { differences in format'. }\end{array}$ \\
\hline $\begin{array}{l}\text { pav:previousVersion skos:narrowMatch } \\
\text { dct:replaces }\end{array}$ & $\begin{array}{l}\text { dct:replaces is a stronger statement (skos:narrowMatch) than pav:previousVersion, as the PAV statement does } \\
\text { not necessarily imply the original was superseded. For instance, a draft specification may be pav: } \\
\text { previousVersion a previously published specification, but it is not dct:replaces the previous version as the } \\
\text { draft is not official yet. }\end{array}$ \\
\hline $\begin{array}{l}\text { pav:previousVersion skos:relatedMatch } \\
\text { dct:isVersionOf }\end{array}$ & $\begin{array}{l}\text { pav:previousVersion is only considered to have a related match dct:isVersionOf, as pav:previousVersion does } \\
\text { not generally cover 'substantive changes in content'. }\end{array}$ \\
\hline
\end{tabular}

of prov:Activity to detail data creation, retrieval, access and publication. Each activity can be associated with prov:Agents which directly or indirectly perform the work. Additional PRV modules allow expression of database queries, HTTP retrieval and file operations, and therefore PRV might at first glance seem like an alternative to PAV. We here consider a use case where Steiner et al. adopted PRV. Below, we explore the complexity of querying the process-oriented PRV approach and we demonstrate how PAV can complement such detailed provenance and simplify queries.

PRV was conceived in 2009, and has been adapted to describe provenance of a range of internet resources, from OpenStreetMap [62] and readings in sensor networks [63] to reified RDF statements [64] and Facebook posts [65]. Here we explore the last case, which presents a browser extension and a REST service for annotating Facebook microposts by combining several natural language processing (NLP) APIs to tag posts with semantic terms from vocabularies like dbpedia.org [66]. The service uses the Provenance Vocabulary (PRV) to indicate how the underlying text mining APIs have contributed to its tagging. This provenance is expressed in rich details of the processes of data creation and multiple data retrievals, including individual API calls, embedding details of their HTTP transactions using the HTTP Vocabulary [67]. An abbreviated example of the resulting tag is included in Figure 16.

The authors of [65] are conscious of the need to reduce the verbosity of their provenance trace, and list this as a consequence of using the PRV vocabulary. We have explored their use of the PRV model and based on their example listing, formulated how one could find out: (i) the APIs called to create the tagging <tag1>, (ii) when the tag was made, and (iii) which agent created the tag. In order to answer this, we have to query through the individual processes of data creation, retrieval and access, as shown in Figure 17.

Using PRV and process-oriented modeling allows the service to express such provenance in detail, but forming this query requires in-depth knowledge about the particular graph structure, which mirrors how the service creates, retrieve and access data. As such, the underlying structure might change significantly if the mechanisms of the service are modified, requiring query rewrites.

The equivalent PAV statements can be queried in a simpler way, as shown in Figure 18. 


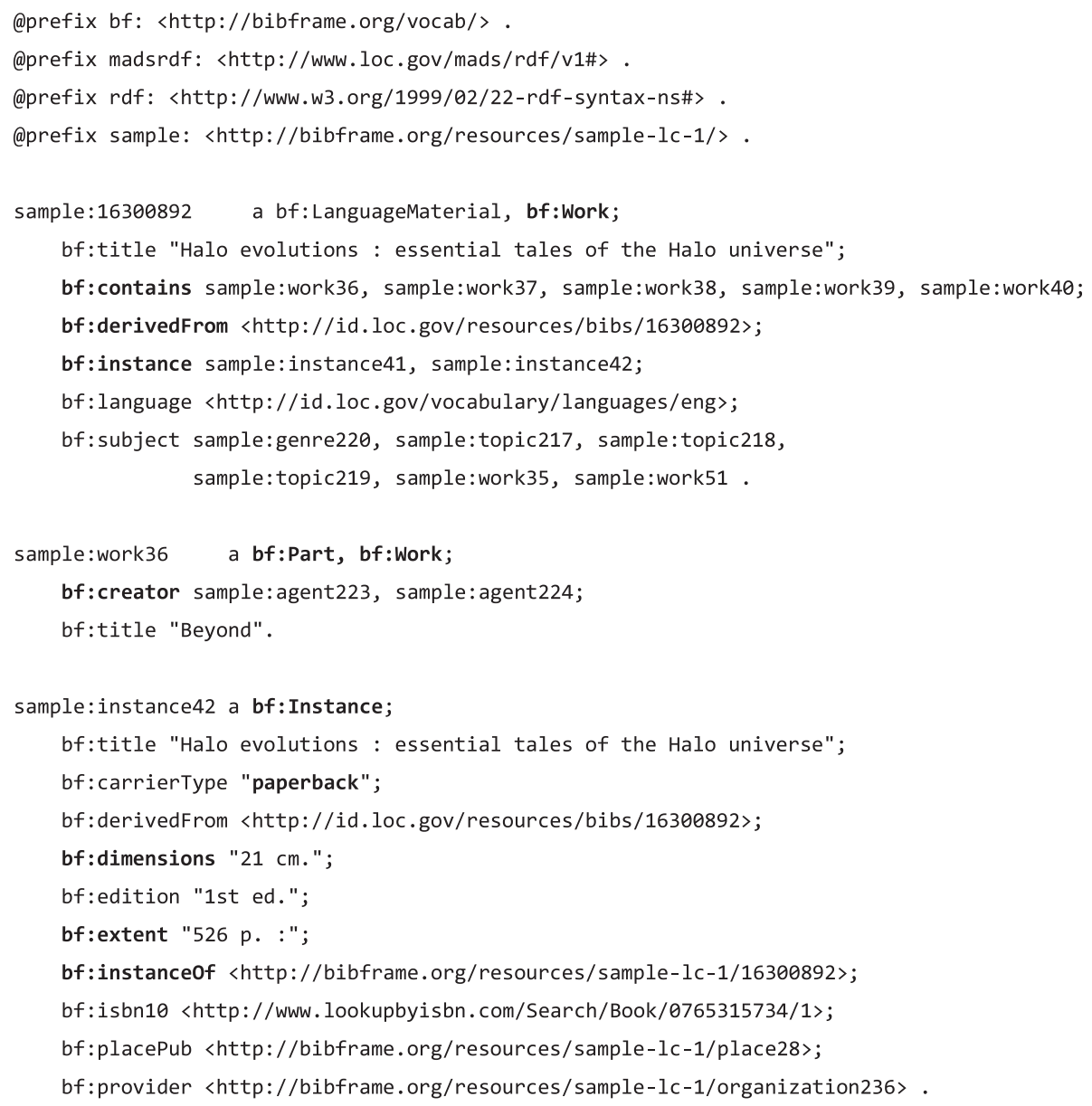

Figure 15 Example of BIBFRAME representation of a book as a creative work (sample:16300892) and its paperback instance (sample: instance42) which have features such as dimensions and pages. Note how this work contains parts (tales) that themselves are works, each having individual bc:creators, and how sample:16300892 (the bibliographical record, not the work) is bf:derivedFrom another bibliographical record. Adapted from RDF/XML example at [60].

We argue that PAV can provide a simpler way to describe provenance from the perspective of the interesting resource. This allows writing general provenance queries without a pre-existing understanding of the specific mechanisms that made the resource.

This simplification does come at a small cost: If multiple? api resources have been imported for the same tag, it is not possible with PAV alone to express when each individual? api was accessed, as import details such as? when and? agent are expressed directly on the resulting resource. We believe this trade-off is reasonable as for common cases there will be a single resource for each of pav:importedFrom, pav:importedOn and pav: importedBy.

Our design decision to not express the implied activities is reflected in all PAV properties such as pav: authoredOn, pav:authoredBy; or pav:sourceAccessedAt, pav:sourceAccessedBy, pav:sourceAccessedOn; and this reflects the simplicity approach of PAV.
This simplicity of PAV's approach does not preclude the concurrent expression of more detailed provenance using other vocabularies such as PRV; as we showed in Figures 18 and 19, more specific details can be expressed by unrolling a PAV statement into a chain of corresponding PROV-O activities and entities. We believe PRV can be used such to compliment PAV for details (and vice versa), and as both ontologies specialize PROV-O without enforcing significant constraints, a PROV-O aware client can follow the traces across both vocabularies; although without gaining the specialized understanding expressed using PAV or PRV.

\section{Conclusions}

In this article we have presented the PAV ontology, a lightweight vocabulary for capturing provenance, authorship and versioning of resources on the Web. PAV distinguishes between the roles of content contributors (including authors and curators) and creators of 


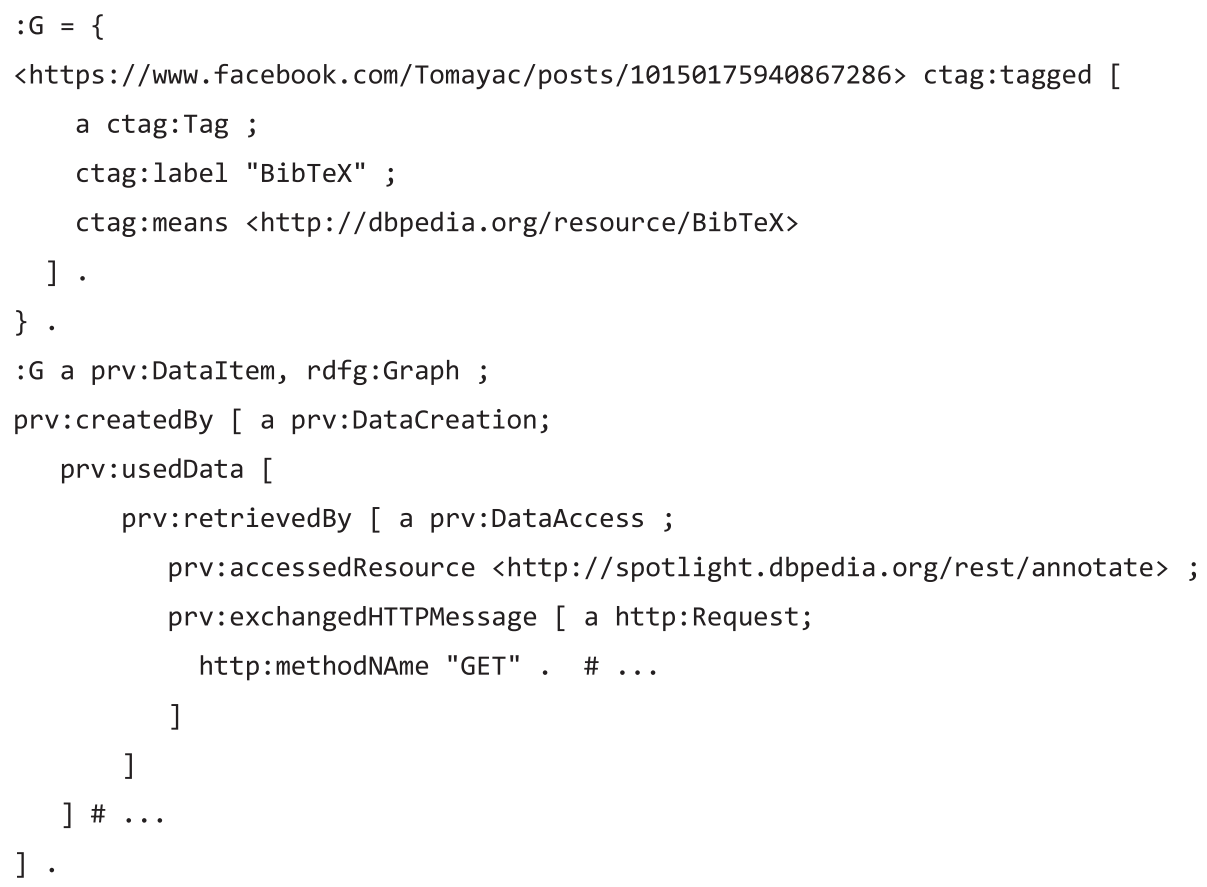

Figure 16 Example of tagging a Facebook post with DBPedia terms using the Common Tag vocabulary [41]. The provenance of the graph that contains the ctag statement expresses a chain of PRV data creation and access activities [61]. In TriG format, abbreviated from figure in [65].

representations. Additionally PAV can describe which sources have been accessed, transformed or consumed in order to create the resource.

As well as the ontology, we have listed examples of projects that have adopted PAV, illustrating their usage through concrete examples. Furthermore, we have presented how PAV extends the $\mathrm{W} 3 \mathrm{C}$ recommendation

\begin{tabular}{|c|c|c|}
\hline \multicolumn{3}{|c|}{ CONSTRUCT \{} \\
\hline \multirow[t]{2}{*}{ ?entity } & prov:wasGeneratedBy & _:import ; \\
\hline & prov:wasDerivedFrom & ?source. \\
\hline \multirow{4}{*}{ _:import } & $a$ & :ImportActivity ; \\
\hline & prov:used & ?source ; \\
\hline & prov:wasAssociatedWith & ?agent ; \\
\hline & prov:endedAtTime & ?when . \\
\hline \multicolumn{3}{|l|}{\} WHERE \{} \\
\hline \multirow[t]{3}{*}{ ?entity } & pav: importedFrom & ?source ; \\
\hline & pav: importedAt & ?when ; \\
\hline & pav: importedBy & ?agent. \\
\hline \multicolumn{3}{|l|}{\}} \\
\hline \multicolumn{3}{|c|}{$\begin{array}{l}\text { Figure } 17 \text { SPARQL query over PRV provenance to find data } \\
\text { creation, retrieval and access of a Facebook tag. The query finds } \\
\text { the activity the tag was prv:createdBy, which was prv:performedBy } \\
\text { the agent and prv:usedData that were prv:retrievedBy another } \\
\text { activity, which prv:accessedResource the given REST API, prv: } \\
\text { performedAt the given time. }\end{array}$} \\
\hline
\end{tabular}

PROV-O, and how this enables detailed provenance traces in PROV-O to be combined with PAV's direct relationships to the origins of a resource.

Originally created in 2006 with curated knowledge bases (such as AlzSWAN) in mind, PAV has evolved and is now used to document a wide variety of digital resources. PAV introduces terms for clearly attributing the intellectual property of the content, and also deals with other aspects crucial for representing scientific content

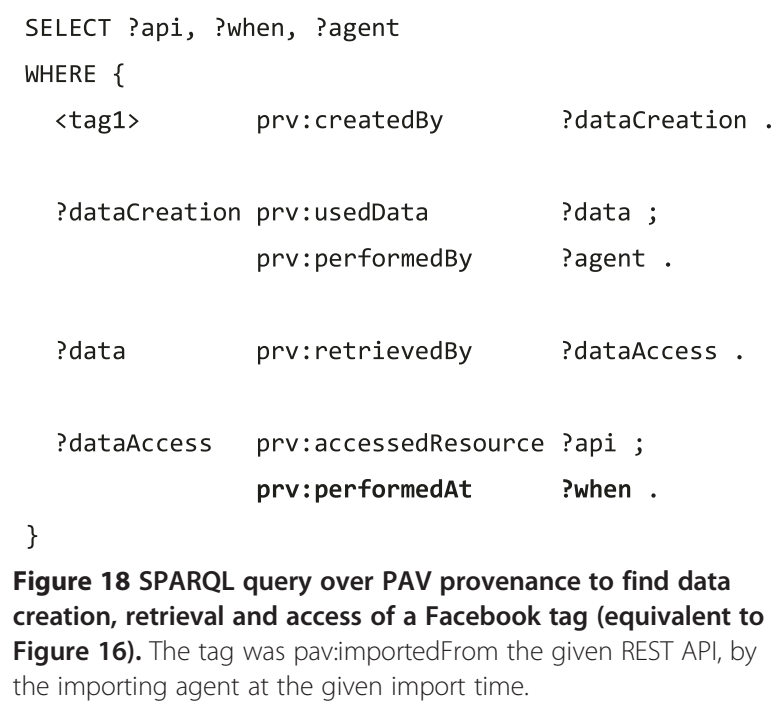

Figure 18 SPARQL query over PAV provenance to find data creation, retrieval and access of a Facebook tag (equivalent to Figure 16). The tag was pav:importedFrom the given REST API, by the importing agent at the given import time. 


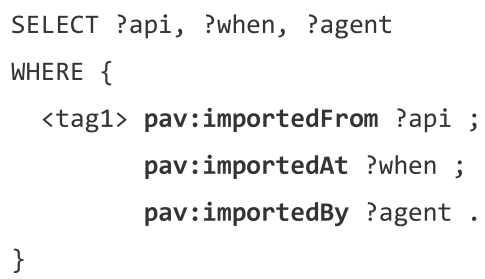

Figure 19 Example of SPARQL CONSTRUCT generating PROV-O activities from PAV imports.

in a federated environment, such as versions and resource retrieval. PAV does not specify detail about the chain of processes that produced the current state of the resource, but gives a view of attribution metadata that is uniform across a multitude of implementations.

At the core of PAV is the distinction between authoring knowledge (content) and creating representations. This is highlighted by the mapping to DC Terms, which shows how PAV properties can provide more precise attributions. Equally important, PAV derivation properties distinguish between plain retrieval, versioned updates, transformational imports, and more structural derivation. These distinctions are essential for attributing resources in the complex real world of curated knowledge bases and datasets, and PAV has been adapted for these purposes by representation models like Open PHACTS dataset descriptions, Nanopublications, Wf4Ever Research Objects and Elsevier's Satellite annotations.

We believe PAV is a good complement to existing provenance vocabularies, such as OPM and PROV-O. Indeed, use of such vocabularies often focus on describing the chain of activities that were performed to transform given resources into other resources, and the role of agents associated with those activities. In PAV, the emphasis is put on the provenance of the resources: PAV describes the lineage from other resources, and just as important, the role of agents involved with creating and maintaining the resource.

\section{Competing interests}

The authors declare that they have no competing interests.

\section{Authors' contributions}

PC conceived and authored PAV 1.0 as a spin-off of the SWAN Ontology. PC co-developed the SWAN platform and is also the main author of Annotation Ontology and the architect of the Domeo Annotation Tool. PC is one of the main authors of this article and co-developed PAV 2.x with SSR. SSR is one of the main authors of this article, and co-developed PAV 2.x with PC. SSR is one of the authors of the Research Object model together with $\mathrm{KB}$, and one of the contributors of PROV-O as a member of the W3C Provenance Working Group. In this article, SSR is the author of the sections on Open PHACTS datasets, Nanopublications, PROV mapping, DC Terms and Provenance Vocabulary. He also maintains the OWL representation of PAV and the accompanying descriptions shown in the Ontology section. $\mathrm{KB}$ is, together with SSR, co-author of the Research Object model and the W3C PROV-O ontology. KB drafted the background and method sections, and contributed to refining the results section. He also participated in improving the descriptions of ontology terms, and by restructuring and improving the organization of the article as a whole. AG is the author of the Open PHACTS dataset descriptions, and helped validate PAV's documentation and OWL implementation. AG co-wrote the sections on Open PHACTS datasets and Nanopublications, and has helped revise the final article. CG is the Principal Investigator of the Wf4Ever project and co-investigator of the Open PHACTS project. She supervised the research and edited the final article. TC is the Principal Investigator of the SWAN project and of the Domeo Annotation Tool. He supervised the research and edited the final article. All authors read and approved the final manuscript.

\section{Authors' information}

Paolo Ciccarese

URL: http://orcid.org/0000-0002-5156-2703

Stian Soiland-Reyes

URL: http://orcid.org/0000-0001-9842-9718

Khalid Belhajjame

URL: http://orcid.org/0000-0001-6938-0820

Alasdair JG Gray

URL: http://orcid.org/0000-0002-5711-4872

Carole Goble

URL: http://orcid.org/0000-0003-1219-2137

Tim Clark

URL: http://orcid.org/0000-0003-4060-7360

\section{Acknowledgements}

We would like to thank Marco Ocana, Gwen Wong, Elizabeth Wu, and June Kinoshita for their input during the development of the SWAN ontology. We also would like to thank Paul Groth for helping us validate the mappings between PAV and the PROV model. We thank the Journal of Biomedical Semantics reviewers, who provided valuable feedback which led to further improvements of the ontology and this paper.

Stian Soiland-Reyes and Khalid Belhajjame are funded for the Wf4Ever project by the European Commission's 7th FWP FP7-ICT-2007-6 270192.

Alasdair Gray received support from the Innovative Medicines Initiative Joint Undertaking under grant agreement number 115191, resources of which are composed of financial contribution from the European Union's Seventh Framework Programme (FP7/2007- 2013) and EFPIA companies' in kind contribution.

\section{Author details}

${ }^{1}$ Department of Neurology, Massachusetts General Hospital, 55 Fruit Street, Boston, MA 02114, USA. ${ }^{2}$ Harvard Medical School, 25 Shattuck Street, Boston, MA 02115, USA. ${ }^{3}$ School of Computer Science, University of Manchester, Oxford Road, Manchester M13 9PL, UK.

Received: 26 April 2013 Accepted: 7 October 2013

Published: 22 November 2013

\section{References}

1. Goble C, De Roure D, Bechhofer S: Accelerating Scientists' Knowledge Turns. In Knowledge Discovery, Knowledge Engineering and Knowledge Management, Communications in Computer and Information Science. Edited by Fred A, Dietz JLG, Liu K, Filipe J. 2013:3-25. doi:10.1007/978-3-64237186-8_1.

2. DCMI Usage Board: DCMI Metadata Terms. 2012. DCMI Recommendation, [http://dublincore.org/documents/2012/06/14/dcmi-terms/]

3. Lebo T, Sahoo S, McGuinness D, Belhajjame K, Cheney J, Corsar D, Garijo D, Soiland-Reyes S, Zednik S, Zhao J: PROV-O: The PROV Ontology. W3C Recommendation; 2013 [http://www.w3.org/TR/2013/REC-prov-o-20130430/]

4. Moreau L, Clifford B, Freire J, Joe F, Gil T, Groth P, Kwasnikowska N, Miles S, Missier P, Myers J, Plale B, Simmhan Y, Stephan E, Van den Busschef J: The open provenance model core specification (v1.1). Futur Gener Comput Syst 2011, 27(6):743-756. doi:10.1016/j.future.2010.07.005.

5. Hartig $\mathrm{O}$, Zhao J: Publishing and consuming provenance metadata on the web of linked data. In Provenance and Annotation of Data and Processes, Third International Provenance and Annotation Workshop, IPAW 2010, Troy, NY, USA, June 15-16, 2010. Revised Selected Papers. In Lecture Notes in Computer Science 2010, 6378. Edited by McGuinnes DL, Michaelis JR, Moreau L. Springer Berlin Heidenberg; 2010:78-90. doi:10.1007/978-3-642-17819-1_10.

6. Gao Y, Kinoshita J, Wu E, Miller E, Lee R, Seaborne A, Cayzer S, Clark T: SWAN: a distributed knowledge infrastructure for Alzheimer disease 
research. Web Semant Sci Serv Agents World Wide Web 2006, 4(3):222-228. doi:10.1016/j.websem.2006.05.006.

7. SWAN Development Team: AlzSWAN knowledge base. Web application. Accessed 2013-10-09. [http://hypothesis.alzforum.org/]

8. Alzheimer Research Forum: AlzForum homepage. Accessed 2013-10-09. [http://www.alzforum.org/]

9. Ciccarese P, Wu E, Wong G, Ocana M, Kinoshita J, Ruttenberg A, Clark T: The SWAN biomedical discourse ontology. J Biomed Inform 2008, 41(5):739-751. doi:10.1016/j.jbi.2008.04.010.

10. National Center for Biotechnology Information: PubMed homepage. Accessed 2013-10-09 [http://www.ncbi.nlm.nih.gov/pubmed]

11. The UniProt Consortium: Update on activities at the Universal Protein Resource (UniProt) in 2013. Nucleic Acids Res 2013, 41(D1):D43-D47 doi:10.1093/nar/gks1068.

12. UniProt Consortium: UniProt homepage. Accessed 2013-10-09. [http:// www.uniprot.org/]

13. W3C Provenance Working group homepage. Accessed 2013-10-09 [http://www.w3.org/2011/prov]

14. Ciccarese P, Ocana M, Soiland-Reyes S: Provenance, Authoring and Versioning (PAV). OWL ontol. Latest version, accessed 2013-10-09. [http://purl.org/pav/]

15. Ciccarese P, Ocana M, Soiland-Reyes S: Provenance, Authoring and Versioning (PAV) ontology v. 2.2. OWL ontol. issued 2013-08-30. [http://purl.org/pav/2.2]

16. Soiland-Reyes S: PAV Ontology changes across versions. Wiki page. Accessed 2013-10-09. [https://code.google.com/p/pav-ontology/wiki/Nersions]

17. Tan W, Madduri R, Nenadic A, Soiland-Reyes S, Sulakhe D, Foster I, Goble CA: caGrid workflow toolkit: a Taverna based workflow tool for cancer grid. BMC Bioinforma 2010, 11:542. doi:10.1186/1471-2105-11-542.

18. Maglott D, Ostell J, Pruitt KD, Tatusova T: Entrez gene: gene-centered information at NCBI. Nucleic Acids Res 2011, 39(suppl 1):D52-D57. doi:10.1093/nar/gkq1237.

19. Prud'hommeaux E, Carothers G, Beckett D, Berners-Lee T: Turtle - Terse RDF Triple Language. W3C Candidate Recommendation. Published 2013-02-19. [http://www.w3.org/TR/2013/CR-turtle-20130219/].

20. Ciccarese P, Peroni S: The collections ontology: creating and handling collections in OWL 2 DL frameworks. Semant Web J. In press [http://semanticweb-journal.net/content/collections-ontology-creating-and-handling-collectionsowl-2-dl-frameworks-0]

21. Hames I: Report on the International Workshop on Contributorship and Scholarly Attribution, May 16, 2012. In , IWCSA ReportHarvard University and the Wellcome Trust; 2012. Accessed 2013-10-09. [http://projects.iq.harvard.edu/attribution_workshop]

22. Shotton D, Peroni S: PRO, the Publishing Roles Ontology v1.5.2. OWL ontology. Published 2013-07-12. Accessed 2013-10-09. [http://purl.org/spar/ pro]

23. Ciccarese P, Ocana M, Garcia Castro L, Das S, Clark T: An open annotation ontology for science on web 3.0. J Biomed Semant 2011, 2(Suppl 2):S4. doi:10.1186/2041-1480-2-S2.

24. Ciccarese $P$, Ocana M, Clark T: Open semantic annotation of scientific publications using DOMEO. J Biomed Semant 2012, 3(Suppl 1):S1. doi:10.1186/2041-1480-3-S1-S1.

25. Schultes E, Chichester C, Burger K, Groth P, Kotoulas S, Loizou A, Tkachenko V, Waagmeester A, Askjær S, Pettifer S, Harland L, Haupt C, Batchelor C, Vazquez M, Fernández JM, Saito J, Givson A, Wich L: The Open PHACTS Nanopublications guidelines v1.8.1. The Open PHACTS RDF/Nanopublication Working Group 2012-03-26. Published 2012-03-26. Accessed 2013-10-09 [http://www.nanopub.org/guidelines/OpenPHACTS_Nanopublication_Guidlines_v1.8.1.pdf]

26. Open PHACTS consortium: Open PHACTS website. Accessed 2013-10-09. [http://www.openphacts.org/]

27. Williams AJ, Harland L, Groth P, Pettifer S, Chichester C, Willighagen EL, Evelo CT, Blomberg N, Ecker G, Goble C, Mons B: Open PHACTS: semantic interoperability for drug discovery. Drug Discov Today 2012, 17(21-22):1188-1198. doi:10.1016/j.drudis.2012.05.016.

28. Gray AJG, Brenninkmeijer C, Evelo C, Goble C, Harland L, Stevens R, Waagmeester A, Willighagen E: Dataset Descriptions for the Open Pharmacological Space. In , Open PHACTS Working Draft. Published 2013-09-19. Accessed 2013-10-09 [http://www.openphacts.org/specs/2013/ WD-datadesc-20130912/
29. Belhajjame K, Corcho O, Garijo D, Zhao J, Missier P, Newman DR, Palma R, Bechhofer S, Garcia Cuesta E, Gomez-Perez JM, Klyne G, Page K, Roos M, Ruiz JE, Soiland-Reyes S, Verdes-Montenegro L, De Roure D, Goble C: Workflow-Centric Research Objects: A First Class Citizen in the Scholarly Discourse. In Proceedings of the Workshop on the Semantic Publishing (SePublica 2012), 9th Extended Semantic Web Conference. Hersonissos, Crete, Greece, May 28, 2012. Edited by Van Harmelen F, García Castro A, Lange C, Good B. 2012 [http://sepublica.mywikipaper.org/sepublica2012.pdf]

30. Kuilman D, Ruck M: Satellites, the Elsevier Format for Ancillary Information to Scientific Journals and Books. In Proc. Int'l Conf. on Dublin Core and Metadata Applications; 21-23 September 2011. Edited by Baker T, Hillman DI, Isaac A, Dublin Core Metadata Initiative. 2011. ISSN 1939-1366 [http://dcpapers.dublincore.org/pubs/article/view/3636]

31. Pence HE, Williams $A$ : ChemSpider: an online chemical information resource. J Chem EduC 2010, 87(11):1123-1124. doi:10.1021/ed100697w.

32. Gaulton A, Bellis LJ, Patricia Bento A, Chambers J, Davies M, Hersey A, Light Y, McGlinchey S, Michalovich D, Al-Lazikani B, Ovrington: ChEMBL: a large-scale bioactivity database for chemical biology and drug discovery. Nucleic Acids Res 2012, 40(D1):D1100-D1107. doi:10.1093/nar/gkr777.

33. Wishart DS, Knox C, Guo AC, Shrivastava S, Hassanali M, Stothard P, Chang Z, Woolsey J: DrugBank: a comprehensive resource for in silico drug discovery and exploration. Nucleic Acids Res 2006, 34(suppl 1):D668-D672.

34. Alexander K, Cyganiak R, Hausenblas M, Zhao J: Describing Linked Datasets with the VoID Vocabulary. . W3C Interest Group Note. Published 2011-03-03. [http://www.w3.org/TR/2011/NOTE-void-20110303/]

35. Brickley D, Miller L: Friend of a Friend (FOAF). Vocabulary Namespace document. Published 2010-08-09. Accessed 2013-10-09. [http://xmlns.com/ foaf/spec/20100809.html]

36. Using Dublin Core Metadata Terms in VolD for general dataset metadata. In Describing Linked Datasets with the VolD Vocabulary. W3C Interest Group Note. Published 2011-03-03. [http://www.w3.org/TR/2011/ NOTE-void-20110303/\#dublin-core]

37. Gray AJG, Hausenblas M: Open PHACTS VoID editor. In, Web application. Accessed 2013-10-09. [http://openphacts.cs.man.ac.ukNoid-Editor/]

38. Groth P, Gibson A, Velterop J: The anatomy of a nanopublication. Inf Services Use 2010, 30(1):51-56. doi:10.3233/ISU-2010-0613.

39. Nanopublications website. Accessed 2013-10-09. [http://nanopub.org]

40. The Biosemantics Group: Gene disease nanopub example. Nanopublication accessed 2013-08-21. [http://rdf.biosemantics.org/examples/gene_disease_nanopub]

41. Iskold A, Mika P, Milicic V, Montgomerie S, Passant A, Taylor J, Tori A: Common Tag vocabulary. Published 2009-06-08. Accessed 2013-10-09. [http://commontag.org/Specification]

42. Callahan A, Cruz-Toledo J, Dumontier M: Ontology-based querying with Bio2RDF's linked open data. Proceedings of the bio-ontologies special interest group 2012. J Biomed Semant 2013, 4(Suppl 1):S1. doi:10.1186/ 2041-1480-4-S1-S1.

43. Miles A, Bechhofer S: SKOS Reference. W3C Recommendation. Published 2009-08-18. Accessed 2013-10-09. [http://www.w3.org/TR/2009/REC-skos-reference-20090818/]

44. Soiland-Reyes S, Bechhofer S, Belhajjame K, Klyne G, Garijo D, Corcho O, García Cuesta E, Palma R: Wf4Ever Research Object Model. Published 2013-08-20. Accessed 2013-10-09. [http://purl.org/wf4ever/model]

45. W3C Provenance incubator group. Accessed 2013-10-09. [http://www.w3. org/2005/Incubator/prov/wiki/Main_Page]

46. Coppens S, Garijo D, Gomez Jose M, Missier P, Myers J, Sahoo S, Zhao J: W3C Provenance incubator group final report. In Edited by Gil $Y$, Cheney J, Groth P, Hartig O, Miles S, Moreau L, Pinheiro Da Silva P. Published 2010-12-08. Accessed 2013-10-09. [http://www.w3.org/2005/Incubator/ prov/XGR-prov-20101214/]

47. Garijo D, Eckert K, Miles S, Trim CM, Panzer M: Dublin Core to PROV Mapping. W3C Working Draft. Published 2012-12-11. [http://www.w3.org/ TR/2012/WD-prov-dc-20121211/]

48. Cheney J, Missier P, Moreau L, De Nies T: Constraints of the PROV Data Model. W3C Recommendation. Published 2013-04-30. [http://www.w3.org/ TR/2013/REC-prov-constraints-20130430/]

49. Wolstencroft $K$, Haines R, Fellows D, Williams A, Withers D, Owen S, Soiland-Reyes S, Dunlop I, Nenadic A, Fisher P, Bhagat J, Belhajjame K, Bacall F, Hardisty A, de la Hidalga A, Balcazar Vargas M, Sufi S, Goble C: The Taverna workflow suite: designing and executing workflows of web services 
on the desktop, web, or in the cloud. Nucleic Acids Res 2013, 41(W1): W557-W561. doi:10.1093/nar/gkt328.

50. Soiland-Reyes S: Visualize PAV provenance as SVG. Taverna 2 workflow, myExperiment. Published 2013-05-01. Accessed 2013-10-09. [http://www. myexperiment.org/packs/418]

51. Sirin E, Parsia B, Cuenca Grau B, Kalyanpur A, Katz Y: Pellet: a practical owl-dl reasoner. Web Semant Sci Serv Agents World Wide Web 2007, 5(2):51-53. doi:10.1016/j.websem.2007.03.004.

52. Moreau L, Dong T, Jewell M, Keshavarz AM: ProvToolbox; Github source code repository. 2013 [https://github.com/lucmoreau/ProvToolbox/]

53. Gray AJG: ChemSpider VoID Descriptor. VoID descriptor in Turtle. Published 2012-08-10. Accessed 2013-10-09. [http://www.openphacts.org/ specs/2012/WD-datadesc-20121019/examples/chemspider-void.ttl]

54. Soiland-Reyes S: SKOS mapping of PAV to Dublin Core terms v0.2.1. SKOS mapping in Turtle. Published 2013-03-01. Accessed 2013-10-09. [http://purl.org/pav/mapping/dcterms]

55. US Library of Congress: BIBFRAME website. . Accessed 2013-10-09. [http:// bibframe.org/]

56. Miller E, Ogbuji U, Mueller V, MacDougall K: Bibliographic Framework as a Web of Data: Linked Data Model and Supporting Services. Washington, DC: Library of Congress. Published 2012-11-21. Accessed 2013-10-09. [http:// www.loc.gov/bibframe/pdf/marcld-report-11-21-2012.pdf]

57. Library of Congress: MARC 21 format for bibliographic data 1999 Edition Update No. 17. 2013. Accessed 2013-10-09. [http://www.loc.gov/marc/bibliographic/]

58. Madison O, Byrum J, Jougulet S, McGarry D, Williamson N, Witt M, Delsey T, Dulabahn E, Svenonius E, Tillett B, John N, Tucker B: Functional Requirements for Bibliographic Records: Final report IFLA Universal Bibliographic Control and International MARC Programme. UBCIM Pub, new series 1997, 19. [http://archive.ifla.org/VIl/s13/frbr/frbr.pdf]. ISBN 978-3598-11382-6.

59. Shotton D, Peroni S, Ciccarese P, Clark T: FaBiO, The FRBR-aligned Bibliographic Ontology v1.7.5. OWL ontol. Published 2013-06-24. Accessed 2013-08-03. [http:/purl.org/spar/Fabio]

60. US Library of Congress: A collection of Library of Congress MARC records representing a small selection of physical resources that have been translated via the BIBFRAME pipeline. Accessed 2013-10-09. [http://bibframe.org/resources/sample-lc-1/exhibit.html]

61. Hartig O, Zhao J: Provenance Vocabulary Core Ontology Specification. OWL ontology. Published 2012-03-14. Accessed 2013-10-09. [http://purl.org/ net/provenance/ns-20120314 ]

62. Keßler C, Trame J, Kaupinnen T: Tracking editing processes in volunteered geographic information: The case of OpenStreetMap. In Identifying Objects, Processes and Events in Spatio-Temporally Distributed Data (IOPE). Edited by Duckham M, Galton A, Worboys M. Belfast, Maine, USA: Workshop at Conference on Spatial Information Theory 2011 (COSIT'11); 2011.

63. Stasch C, Schade S, Llaves A, Janowicz K, Bröring A: Aggregating Linked Sensor Data. In The 4th International Workshop on Semantic Sensor Networks 2011 (SSN 2011), Workshop of the 10th International Semantic Web Conference (ISWC 2011). CEUR Proceedings 839. Edited by Taylor K, Ayyagari A, De Roure D. Bonn, Germany: Aggregating Linked Sensor Data; 2011.

64. Quasthoff M, Meinel C: Tracing the Provenance of ObjectOriented Computations on RDF Data. In Proceedings of the Second Workshop on Trust and Privacy on the Social and Semantic Web (SPOT2010). Edited by Kärger P, Olmedilla D, Passant A, Polleres A. Heraklion, Greece: CEUR-WS.org; 2010.

65. Steiner T, Verborgh R, Gabarró Vallés J, Van de Walle R: Adding meaning to Facebook microposts via a mash-up API and tracking its data provenance. In Next Generation Web Services Practices (NWeSP), 2011 7th International Conference on Next Generation Web Services Practices. Edited by Abraham A, Corchado E, Han SY, Guo W, Corchado J, Vasalikako A. Salamanca, Spain: IEEE; 2011:342-345. doi:10.1109/NWeSP.2011.6088202.
66. Sören A, Bizer C, Kobilarov G, Lehmann J, Cyganiak R, Ives Z: Dbpedia: A nucleus for a web of open data. In , The semantic webHeidelberg: Springer Berlin; 2007:722-735.

67. Koch J, Velasco CA, Ackermann P: HTTP Vocabulary in RDF 1.0. W3C Working Draft. Published 2011-05-10. Accessed 2013-10-09 [http://www.w3. org/TR/2011/WD-HTTP-in-RDF10-20110510/

doi:10.1186/2041-1480-4-37

Cite this article as: Ciccarese et al:: PAV ontology: provenance, authoring and versioning. Journal of Biomedical Semantics 2013 4:37.

\section{Submit your next manuscript to BioMed Central and take full advantage of:}

- Convenient online submission

- Thorough peer review

- No space constraints or color figure charges

- Immediate publication on acceptance

- Inclusion in PubMed, CAS, Scopus and Google Scholar

- Research which is freely available for redistribution

Submit your manuscript at www.biomedcentral.com/submit
() Biomed Central 